$$
2 n-841
$$

ORNL -4969

\title{
MATHEMATICAL DESCRIPTION OF FISSION PRODUCT TRANSPORT IN COATED PARTICLES DURING POSTIRRADIATION ANNEALS
}

R. B. Evans III

M. T. Morgan 



\begin{tabular}{|c|c|}
\hline & 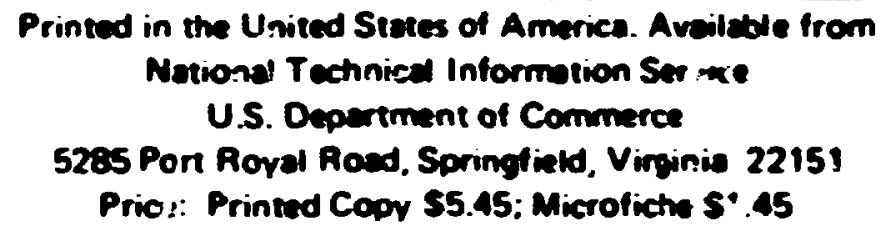 \\
\hline
\end{tabular}

This report we preperid es en sccount of mork sponsored by the United

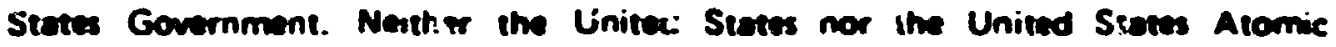
Enerey Cormmission. wer any of their moloyess. nor sny of thair contractors. subcontrectors, or the:- imployess, makes any verrenty. exporizs or imolind. or sumes any her lisbility or responsibility for the a urec., ecmsteteness of usefulness of eny informotion. apperatus, product if mocess isielosed. or represenss thet iss use would not infringe privately owred righis. 


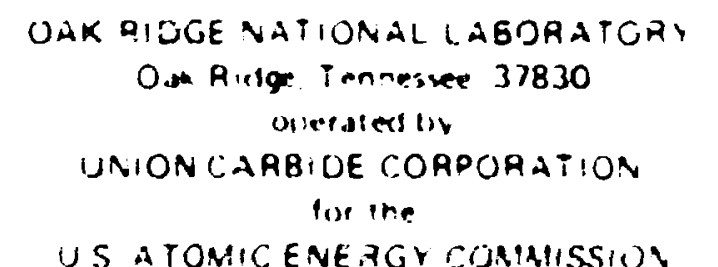

US A TOMIC ENEAGR CONASISSIOU 


\section{CONTENTS}

Abstrat $\ldots \ldots \ldots \ldots \ldots \ldots \ldots \ldots \ldots \ldots \ldots \ldots \ldots \ldots \ldots \ldots \ldots \ldots \ldots \ldots \ldots \ldots \ldots$

1. Introduction $\ldots \ldots \ldots \ldots \ldots \ldots \ldots \ldots \ldots \ldots \ldots \ldots \ldots \ldots \ldots \ldots \ldots \ldots \ldots \ldots \ldots \ldots \ldots \ldots$

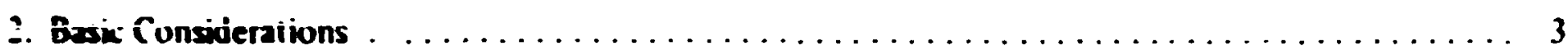

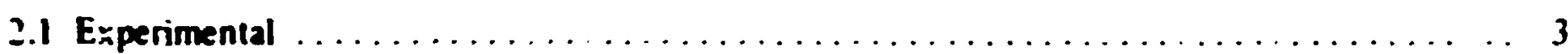

2.1.1 The Diffusion Experiment $\ldots \ldots \ldots \ldots \ldots \ldots \ldots \ldots \ldots \ldots \ldots \ldots \ldots \ldots$

2.1.2 Sume Diffuion Results $\ldots \ldots \ldots \ldots \ldots \ldots \ldots \ldots \ldots \ldots \ldots \ldots \ldots \ldots \ldots$

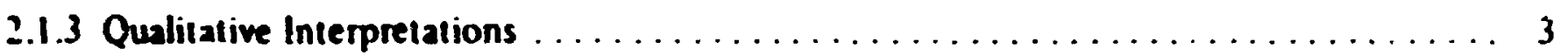

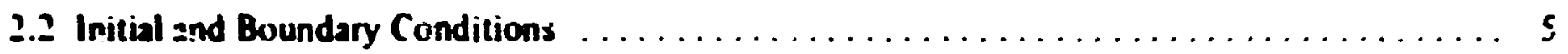

2.2.1 Prelininary Remarks $\ldots \ldots \ldots \ldots \ldots \ldots \ldots \ldots \ldots \ldots \ldots \ldots \ldots \ldots \ldots \ldots$

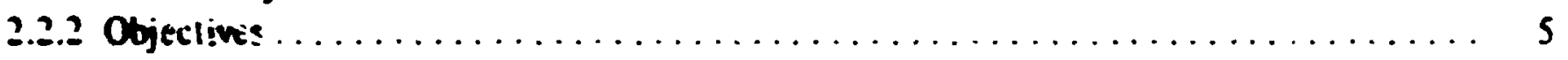

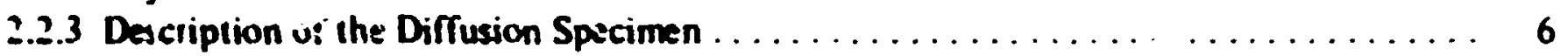

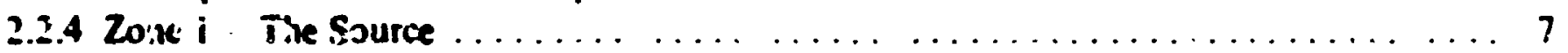

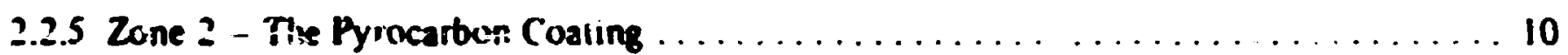

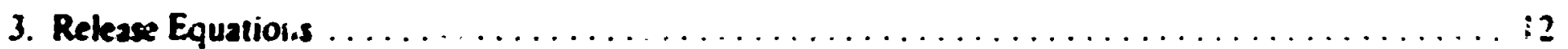

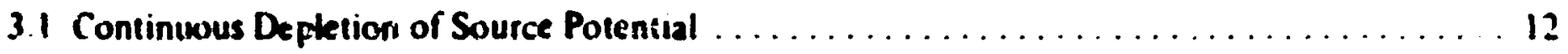

3.1.1 Radial Flow in Spherical r widinates $\ldots \ldots \ldots \ldots \ldots \ldots \ldots \ldots \ldots \ldots \ldots \ldots \ldots \ldots \ldots$

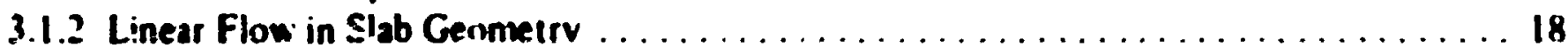

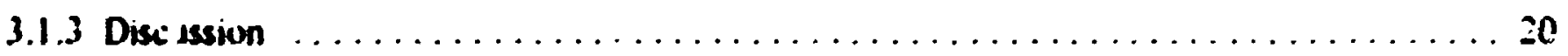

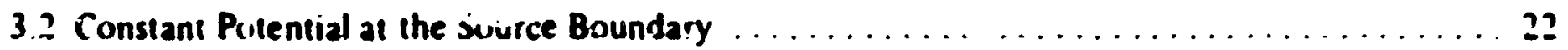

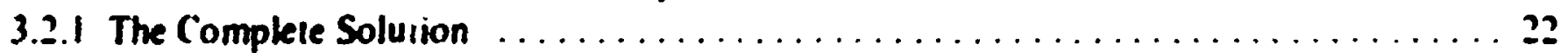

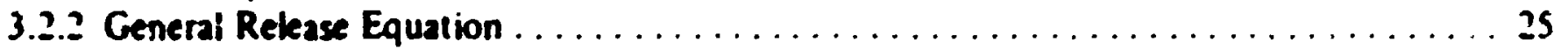

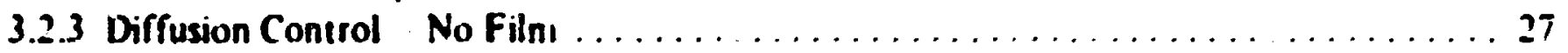

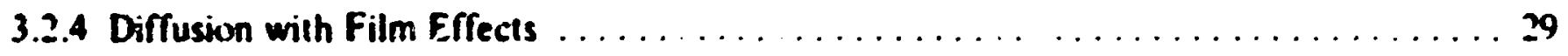

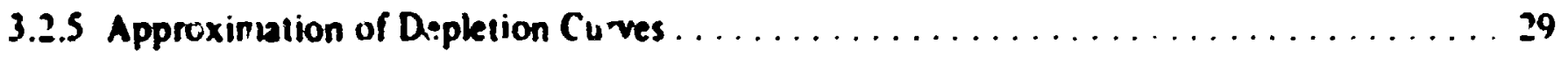

3.3 Depletion After Constant.Potential Operation $\ldots \ldots \ldots \ldots \ldots \ldots \ldots \ldots \ldots \ldots \ldots$

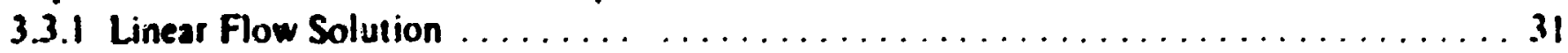

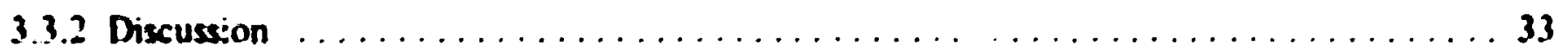

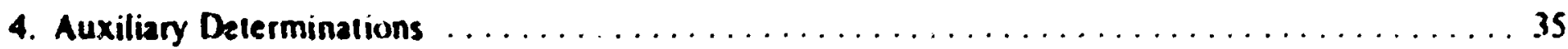

4.1 Experiments with Separated Particle Components $\ldots \ldots \ldots \ldots \ldots \ldots \ldots \ldots \ldots \ldots \ldots$. $\ldots \ldots \ldots$

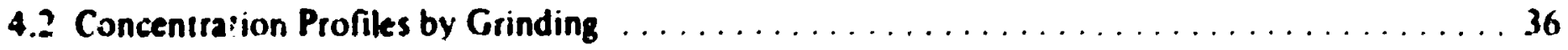

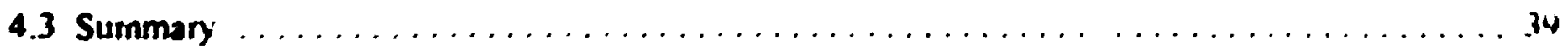

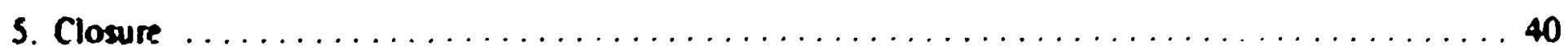

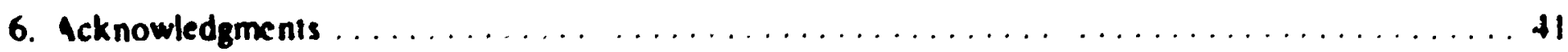

7. Nometrclature $\ldots \ldots \ldots \ldots \ldots \ldots \ldots \ldots \ldots \ldots \ldots \ldots \ldots \ldots \ldots \ldots \ldots \ldots \ldots$

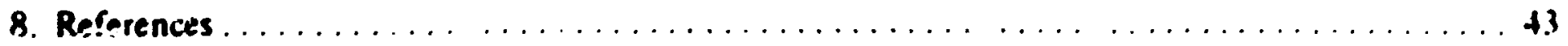




\title{
MATHEMATICAL DESCRIPTION OF FISSION PRODUCT \\ TRANSPORT IN COATED PAKTICLES \\ DU:ING POSTIRRADIATION ANNEALS
}

\author{
K. B. Evans !II M. I. Morgan
}

\begin{abstract}
This repurt is cuncerned with s:-reral integated forms of the diffusion equation that might be used tu intsrpret and curreble cesium and perhaps stron:ium) release dab obtained from postisradiatinn anneals of : inrocarbon-cuated fuel partictes. Emphasis is miced on the role of the initial and boundery crinditions as influenced by cource concentration kevek. permanent trapping in the source, and partitiun cuefikients berween the source and coating It is assumed that release rates are controlled by the usurce pote.lial and the diffusiviy of the coating (with and without an external fitm or evaporatiun coeffiniert). A eeriew of the equations. coupled with considrations of corredation protedures, indicates the need for cumplementary experiments that vill give independeat measurements of the trapped isctixn and boundary concentrations.
\end{abstract}

\section{INTRODUCTION}

Depusiled cesium isotupes art responsible for most of the gamma activity in STCR primary coolant circuits exannined eu date: ${ }^{1.2}$ moreuver. it is clear that release of cesium into the frimary circuil should be contrulled for the following reasons:

(1) The extent of internal cesium! contamination will guvern access limitations which. in turn. will deternine the feasibility and/or costs of mairiziance following shuldown.

12) Loss of the helium coulant. which contains traces of cesium. during a blowdown accicient could intluence subsequent cleanup and land use costs. even through short-term doses 10 individus? at site beundaries are of lisile significance.

Cesiem released iron fuel particles is delayed to a certain extent in structural graphice iomponents suppurting and surrsunding the iuel cumpacts, but our knowledge about the mobility of cesium uncer such circumstances is liamited. Foven a rate of release from the fuel. one would be hard pressed to make a re able estimate of the anount of ce ium that would permeate the fucl steeve during a four-year fuel cicle. Any benefits of delay nughi even valish when ene cunsiders gas turbine itTCRs at very high temperalures. Thereforc. cesium cuntrol must rely on the retention efficiencies of the fuel particles. A prime objective of this study has ocest cullection of infurmation which will aid in determination of the degree of control to be expected frum particles cur:pprising the fertile compunent of the cursent reierence fuet." Aside from these bruad considerations. uur immediate areas of cunceon may the intriduced by iracing fuel particle devekupment wrer the last decade Irum tix standpoint of the authars' involvement.

karly relerence desagns for luel particies were based un LC, paiticles couled with either lamellar or

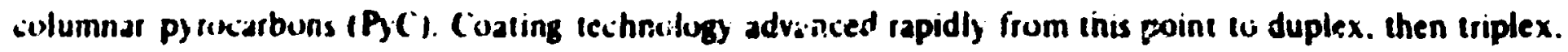
and linally to vartous arrangenkellis imiluding buffer tajers. Fo: the imltial configinations. It was found that nuble gases were well cuntanned but that nelals such as barium. cesium. a id others would leak through the

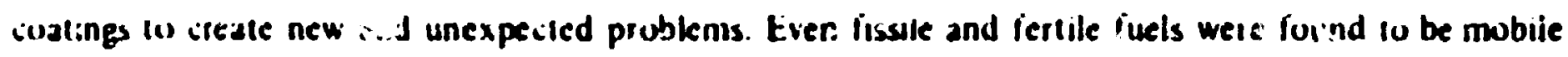

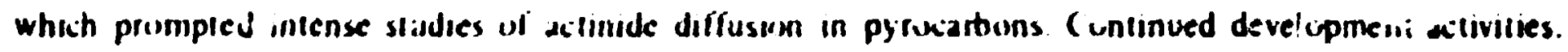
huwever. revealed that acinnick migration ciculd be effecilively cilminaled by using oxide. rather than sarbide. kernels. The problem of migration ci lissu in products of imtermediate volatiliry (e.g. cesium) resiained. and thiss could only be eliminatad by the use of improved silicion cartide cualings. which also held up aclunides. 
Current It

in the United Stats) fissile particles are UC ${ }_{2}$ kernels with Buffer/PyC/SiC/PyC cuating layi on one concept, the fertile particles might be $\mathrm{ThO}_{2}$ with Buffer/PyC layers (called oxide - BIS(S). It is postulated that the BISO coating could be used to enhance fuel fabrication and repracessing economy. If this concept proves inadequate, one could always revert to the coating, array on fissile particles. Our present problem involves evaluation of the retention performance of the oxide-BISO concept as it applies to fartile particles.

Considerable research effort has been expended boti, at ORNL and GAC on siut::; of :; slum migration ihroupt: coatings on BiSO-oxide particles. The initial philosophy behind local offort: centered on relative reiintion efficiencies of various coatings. Five years ago it was sufficient to perform a reiease experiment,

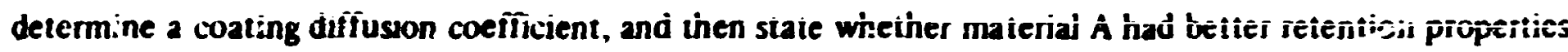
than material B (i.e., a lower coefficient). However, extensions of this work have forced the realizatica th.dt more than a diffusion coefficient is required to specify release 0 : retention properties. In fact, it now appears that. although cesium relesse might be predicted in tems of classical diffusion (heat transier) models. additional considerations relating to irreversible trapping nay be required. This is of considerable importance since a large portion of the BISO effort can be salvaged in the event that we are forced to abanden the BISO concepr in lieu of the less imaginative, more conservative TRISO concept; the latter ircludes, of co irse, the SiC layer." The portion tivat can be salvaged includes the careful delineation of various effects nelative to kernels, buffer layers, and outer contings that might permit translation of present results to more "aoianced" con xepts.

This report is one of two concerred with the collection of theoietical and experimental material relative to cesium relea: = iron: BISC.-cuated oxide particles. Specifically, this report deals with the various equaiions derived as boundary-value problems applicable to cesium ieiease experiments. For a first apricication. some of these equations will be applied to completed experiments in order to extract a maxirnum amouni of information from available data. For a second applization, they will be used for the design of supplementary experimente with materials studied to date. The success of these applications will be detailes in subsequent reports.

Several excellent reviews of equations that describe release rates of fission produrts from irradiated fuels are given in the literature. The most definitive of these relate to noble gases. When one seeks mathematical descriptions of the mechanisms controlling cesium release from intact B'iSO particles, the amount of available infonnation is very limited. Theoreticians move rapidly to extensioins covering reactor operating conditions. where production and de:ay phenomena must be accounted for at the expense of other. seemingly less dominant, effects. Conditions applicable to postirradiation anneals of the type we f-rform are quickly passed over in a search for generality. A report most pertinent in application to present

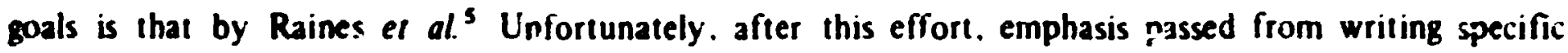
equations in closed for.. in computerized numerical solutions of heat cquations that take into account numerous possible complexities. Examples of this new approach ar: given by Dunlap and Gulden.

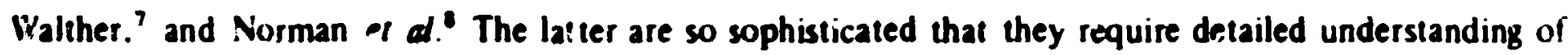
input programs that may or may not apply to our problems. To avoid a review of computer techniques. we decided to use a direct approach to solving the problems. We would try to select woll-known squations that have worked well in the past for correlation of transport behavior. This report deals with a discussion of the selections and the significance of the initial and boundary conditions involve: from an experimental point of view. 


\section{BASBC CONSIDERATIONS}

\subsection{Experimental}

\subsubsection{The Liffusion Experiment}

Siveral kinds of lanoratory determinations and auxiliary experiments are employed in studies of fission product release from coalce fuel parlicles. but pustirradiation anneals of intact particles conscituis the heart of the entire experimental progesm. If the irradiations are conducted at low temperalures $i<800^{\circ} \%$ )

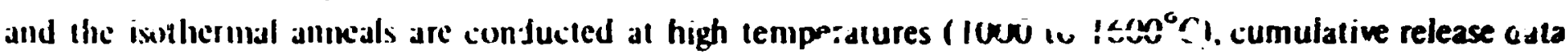
obtained during the anncals provide delinitive information concerning the release mechanism becawse some izedsitre of iuntro! wi!! !have been exercised over the initial and boundary concentrations within the particles

The diffusion anneal experiments condicted to date have specialized in studies of ${ }^{13} \mathrm{C}$ s release, with particular emphasis on BISO oated oxide fuel particles. In these experiments, irradiated particles are analyzes for initial ${ }^{137}$ Cs colitent isy gamma-ray spectrometry (total cesium, including ${ }^{133} \mathrm{Cs}$ and ${ }^{135} \mathrm{Cs}$, (a)i be estimalted): then they are heated for predetermined time intervals at an assigned constant temperature. The contigurations and environment within the high-temperature containn.ent envelope are arranged and adjusted such !hat all the cesium released as a vapor during an anneal int,rval will condense on cool collaction surfaces adjacent to the hot zone abcut the particles.

To separate gamma-ray contributions from released and retained cesium, the furnace is shut down after each anneal interval. and individual gamma analyses are performed on all components of iaterest. The cumulative buildup of cesium on collection surfaces is utilized as a measure $\boldsymbol{x}$ the tiaie-integrated release rates because release values are much smaller than retained va!ues. Additional details are available elsewhere.

\subsubsection{Some Diffusion Results}

The $1600^{\circ} \mathrm{C}$ iclease behavior observed during anneals of twe types of BISO-coated $\mathrm{TI} . \mathrm{O}_{2}$ particles is illustrated in Fig. 1. Here the cumulative cesium release data are plotted as a function or time, and the release values are expressed as fractions of the total cesium present at the beginning of the experiment. Such pluts are uften desigrated as iractional release curves. Both curves illustrate one of the more impressive aspects of the experiments in that a period of well over two months was required for their completion. (This includes shutdown times to perform the gamma analyses.)

Curve II is typical in the sense that the experiment was terminated before a fractional release of 0.20 was attained. Curve $I$ is atypical, and in fact unique, because these are the only ORNL intact particle results demonstrating that the ultimate release values could be considerably less than $100 \%$ of the initial content. Similar La: a prooably cculd have been obtained for particles of curve II, but an additional six months of experimentation might have been required. Aside from indications of an ultimate release vaiue. both curves reveal iommon features which would have been more apparent if the ordinaie values for curve II had been plotted on an expanded scale.

\subsubsection{Qualitative Interpretations}

Most sets of data involving extended anneal times have features common to regions $A$ and $B$ : several show region $C$ characteristics. Regions $A$ an $1 C$ correspond 10 lime regimes whercin transient conditions prevail within the particle. Throughout region $A$. the coating is loading up with cesium: over $C$. is would appear that the driving force at the source boundary is falling which. in turn. permits the coating to unlozd. 


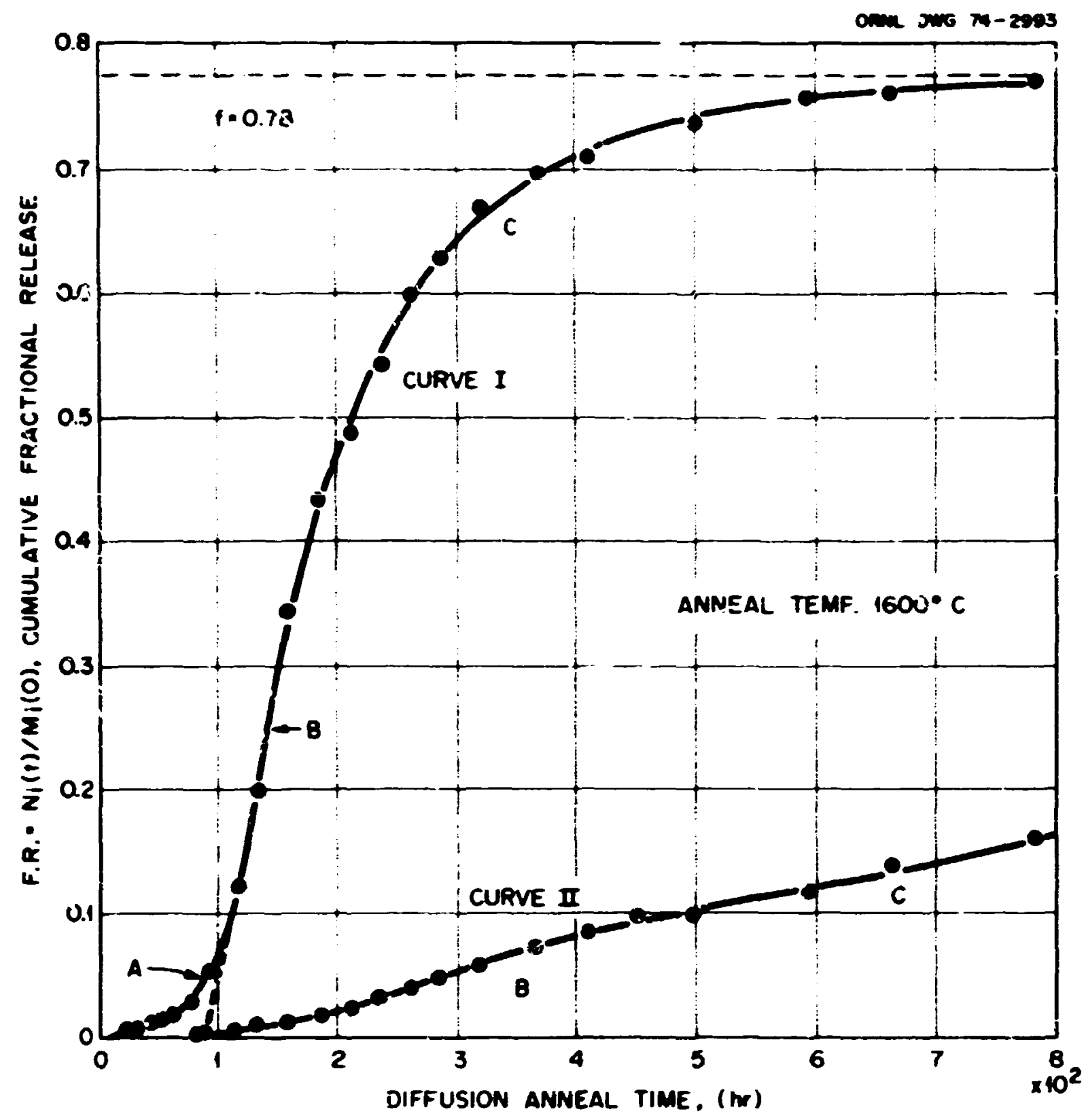

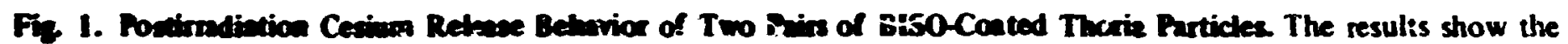
rebtive retention effectiveness of LTI coaings derived from propylene (Curve I) and HTI coatings derived from methane (Curve II).

The intermediate region. B. seerr: :o represent a quasi-steady state regime in which release rates are nearly conc:ant with time. Linea: ex.trazolation si the region B segment to the time axis gives a "breakthrough time," which can be described as thic ume that cesium would first appear outside the coating if the material had moved through the coating uniformly without dispersion. It is standard practice to evaluate an admit!ance pirameter on the basis of the breakthrough time and, in the absence of auxiliary information, t. $c$ cali it a diffusion coefficient. Although the validity of this approach seems questionable, we will show that it gives surprisingly good approximations in many nonideal cases.

Another important feature of the release curves relates to the slope in region B. Since this slope is nearly constant, it should reflect the influence of a stead'state rate enuation that carries contributions of a driving potential, the diffusion coefficient, and (unfortunately in sume cases) additional parameters representing subrle side effects. If the latter were negligible, orie could - iin principle - extract iwo values of importance fro $n$ data in region B, namely, a diffusion coefficient and a driving potential. This is precisely the approach one would take for systems exhibiting a time-constant potential at the source butndary and a zero potential at the outer boundary.

The crux of the matter reduces to whether: (1) all three regions are governed by three distinctly different mechanisms, (2) one mechanis 7 applies for regions $A$ and $B$ and a second for region $C$, or (3) a 
single mechamism controis all regiontis. The last possibility must be included because the curve for this case will exhibit matrifestations of all three regior s. An examimation of the curves ori Fig. I reveals that a Inechar is:ic resolution cannot be obtain a through a ci sory inspection of a release curve: the time boundaries cannot be distinguished. To accomplish this. one must compare either the entire curse or expanded portions of the curve 10 solutions of the diffusion equations detiyed under various conditions. These conditions should represelll reasonable linir ing cases. The selection and discussion of sich equitions are prime sbjectives of this report. We shall start with a gineralized dixiusion of the conditions that set the form of the equations.

\subsection{Initial and Boundary Conditions}

\subsubsection{Pretiminiary Reningks}

For purposes of orientation we shall briefly describe a fuel particle and its components. A BISO-coated oxide particle has three major coniponents: a thorium or uranium oxide kemcl. a buffer layer, and an outer pyrolytic carbon coating. The kernel is usually a high-density material; the buffer is a very porous iarbonaceous material designeri (") accommodate dimensional ch?nges and absorb recoil damage; the outer coating is a moderate-density is "tropic p.roca; oon designed to serve as a tiny leakproof pressure vessel. In addition. earlier specifications required the presence of a thin extemal coating (columnar PyC) ostensibly to avoid fabrication damage when a laige number of particles were bonded together to form a fuel stick. Most of the ORNL experiments have involved particles witi such "sacrificial layers" simply because they were available as large batches of identical materials.

Since niany of t' e particles have been subiected to high burnup levels prior to diffusion anneais, it is pertinent 10 describe the physica: condition of the particles after irradiation. It turns out that diffusion annealing at moderately high temperatures does not alter the appearance of the particle. Therefore, wr, have elected to discuss one that was irradiated to $20 \%$ bu:nup (FIMA) and then annealed at $1250^{\circ} \mathrm{C}$ for $2500 \mathrm{hr}$ 11 obtain a fractional release of 0.25 . Figure 2 gives a pictorial representation of a photomicrograph of a water-polished secrion. along with two microprobe scans of the same section.

Two interpretive restrictions on microprobe results should be mentioned here. First, ratios of average sian-recorder readirgs for two different matrices are not equivalent to the actual average concentration ratio. The scan values on the ordinates of Figs. 2(b) and 2(c) only reflect relative cesium concentrations within a given region. Fortunately. total cesium. as seen by the probe in a given region. is direclly proportional to the tracer isotope ${ }^{137} \mathrm{Cs}$. which forms the basis for our diffusion data. Total cesium is present as ${ }^{133} \mathrm{Cs} .{ }^{135} \mathrm{Cs}$, and ${ }^{137} \mathrm{Cs}$. The first two are stable: the tracer is long-lived. Thus the ratio of ${ }^{137} \mathrm{Cs}$ to total cesium remains constant at $\sim 1 / 2$. This is ine of the favorable features of cesit:m experiments.

The second restriction on microprobe results involves false concentration decreases, or sudden "dips" in the scan readings. These oucur when the probe encounters deep voids. Probe emissions are lost at these points. and the detector will interpret such losses as decreases in cesium concentration. Some perspective regarding the interpretation of scans may be gained by noting the probe reactions to the mounting plastic beyr. $d$ specimen edges. as shown in Figs. 2(a) and 2(c).

\subsubsection{Objectives}

One might question the wisdom of placing emphasis on limited microprobe data which are only, quilitative at the preserit stake of developmeni. A respronse would be to point out ihat the selection of appropi:ate release equatiriis requires knowledge about internal concentration profiles. as, well as internal conditions governing the shape of the profiles. Such information cannot be extracted froni current ORNL 


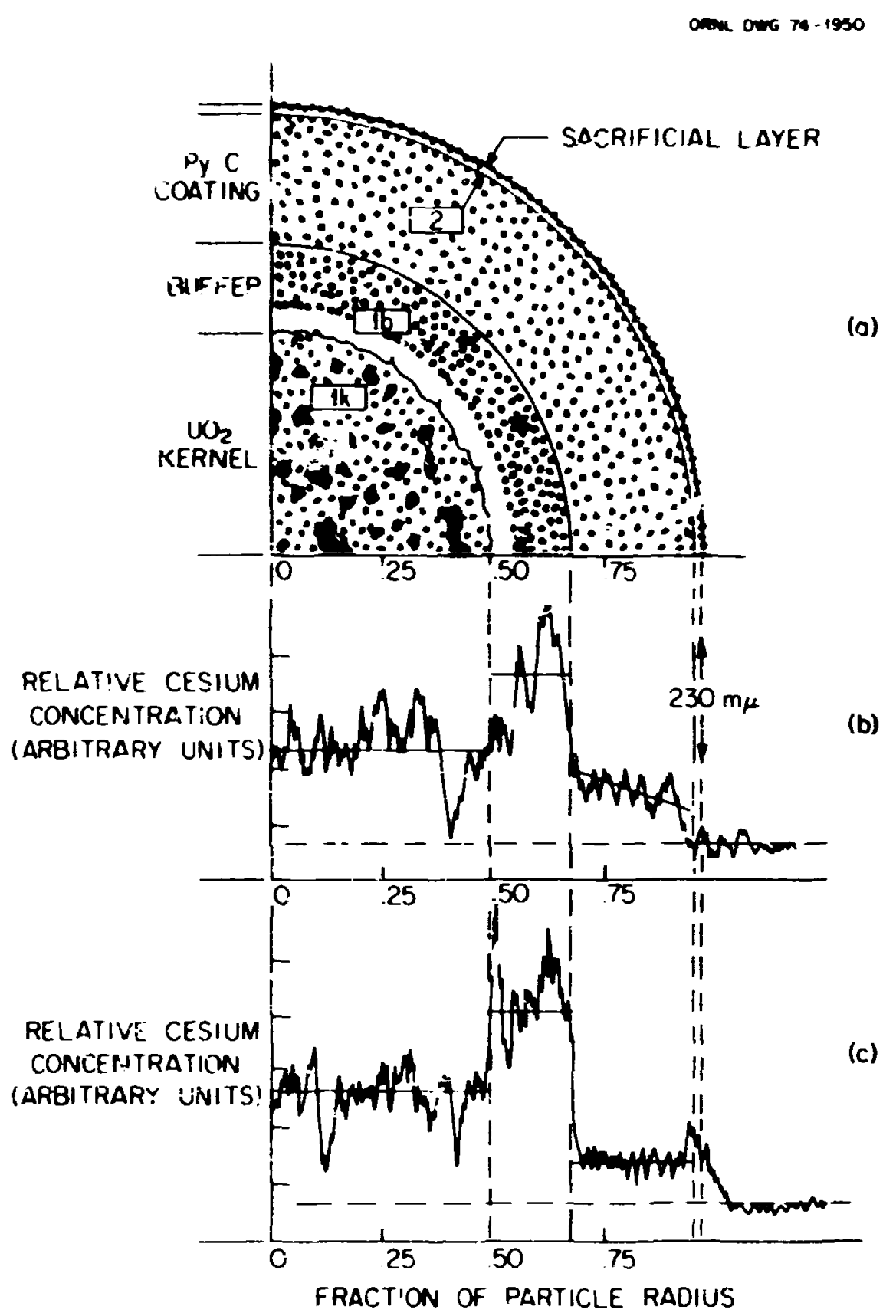

Fig. 2. Microprobe Data Obtained bv Scanning Components of a BISO-Coated UO, Particle. Prior to scanning. this particle was irradiated to $20 \%$ burnup. annealed at $1250^{\circ} \mathrm{C}$ for $2500 \mathrm{hr}$ to $25 \%$ fractional release, and then sectioned and polished.

data. The latter relate to external measurements of cumulative release; there is no way to backtrack and acquire the required information. For the time being, the only recourse is 10 use the microprobe data, with some degree of skepticism, for resolution of the following points: (1) location and definition of a source zone as it appears after an appreciable diffusion-anneal time; (2) examination of diffusion resistances within this zone; and (3) extrapolation of information for (1) and (2) from extended times to earilier periods. in order to obrain estimates of the initial conditions.

Considerations of point (3) require heuristic speculations and will be discussed in more detail in a subsequent section concerning phenomena in the source zone. It should be remarked here that considerable att.ntion is given to boundary conditions but that oueries relative to initial conditions are of:en ignored. This is understandab!e since experiments designed to establish initial conditions tend to identify all the weaknesses in laboratciry procedures.

\subsubsection{Descripsion of the Diffusion Specirien}

The Kernel. Irradiations to high burnup levels produce dramatic effects in both the kernel and buffer regions. Large voids appear in the initially dense or.ide kernels. Exhaustive microscopic examinations reveal 
that void stess increase as burnup increases. and that voids tend to merge during growth. Since significant amoumls of cesium moved out of the kernel during migration. it must be assumed that many vosids are interconnected. We note that now is the only criteiton available for the degree of connection. Pore comncetions can rever be pinpointed via twe-dimensional microsiopy.

Bused on the microprobe results. long-term cesium drainaga did not produce a concentration decrease over the kernel region. Resistances in the kernel did not control release rates. However, an appreciable fraction of the fission products was irnmobilized in some mysterious fashion. In view of the damage suitered by the kernel. it seems logical to assign microscopic volumes within the kernel as the region of residence for trapped fission produ:ts.

The Buffer Layer. Tha original leaturcless structure of buffer matiial (actually a cheas soot,

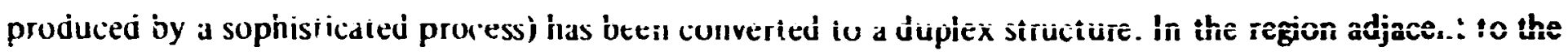
kernel. severe alter:ations resulting from recoil and knock-on phenomena have occurred. In simpler terminology. the buffer here is "irammed full" with heavy fuei and solid fission produsts. Th.e microprobe detects high cesium concentrations at the outer, more porous. buffer region. This segregation probably resulted from an overpopulation syndrome the cesism had nowhers slse to wait before its transport out through the coating. A literal translation of microprcbe ciata for the tuffer region wouid suggest movement into the particle; an "honest interpretation" would yield a pcsitive slope. The need for skepticism is hereby demonstrated; it is known that cesium moved out of the particie. We no:e also the formation of precipitates and of new voids in the buffer region. While it is difficuit to assess their effects, they could have marked intluences on the microprobe scans.

The Pyrolytic Carbon Coating. - The condition of the outer coating is remarkably unchanged at the mag. if ications involved $(\sim 3) 0 \times)$. Nearly all damage extending beyond the kernel is absorbed by the buffer. Voids in the coating are no more pronounced than they were prior to irradiation; thev remain as isolated voids. As to cesium profiles. nothing definite can be attributed to the presence of the sacrificial layer. The first microprobe scan over the coating exhibits a definite concentration decrease; the second does not. An average of a large number of scans, however, would probably have a definite negative slope, verifying the coating's ability i’l act as a diffusion barrier.

Summary. -- The prsive data support a!! natural impulses to fix the outer boundary of the sulurce zone as the buffer layer-PyC coating interface, and to homogenize the kernel and buffer regions (lk) and (lb) into a single source zone. Although regional concentrations within the source zone are different when referred to bulk volumes, it will not be difficult to concoct an average concentration for the source because diffusion resistarices in regions (lk) and (lb) appear small in comparison to those envisioned for the coating. Thus we have the advantageous situation in which we combine ali unknown st.uctural effects related to damage and trapping in the source zone and divorce them from the diffusion parts of the problem. The trapped fraction can be dumped in a negat $\%$ potential well. Finally, we note that the shapes of microprobe traces are quite similar to hypothetical profiles used to derive the diffusion equations that will appear in subsequent sections.

\subsubsection{Zone 1 - The Source}

Discussions of the experimental procedures emphasized the role of cesium vapror transport as a key factor in external release measurements. Since formation of reduced cesium vapor is farnred at high temperature. it is felt that a gas transport mechanism also controls cesium migration in the porous regions of the source zone. We envision that the cesium will attain some partial or vapor pressure, $p^{\prime}=n^{\prime} T^{\prime}$, as dictated by chemicial potentials within the kernel and/or buffer. where the primes denote values corresponding to mobile cesium. Then we can write for the initial content, using subscripts $k$ and $b$ for 
kernel ind buffer regions:

$$
\left.V_{k}\left|\epsilon_{k} n_{1}^{\prime}(0)\right|+V_{b} ! \epsilon_{b} n_{1}^{\prime}(0)\right]=M_{i}^{0}
$$

Gas densities are referred to pore volumes; the porosities, $\epsilon$, convert these to bulk volumes, $V$. The $\mathrm{AM}_{1}^{\text {? }}$ denotes the total amount of mobile cesium available initially. All pore volumes over $k$ and $b$ are assumid to be connected, and the gas density (pressure) is to be uniform throughout the source. The gas density is, therefore. given by:

$$
n_{1}^{\prime}(0)=\frac{n M_{1}^{0}}{\Sigma V_{p}}
$$

where

$$
\Sigma V_{p}=\epsilon_{k} V_{k}+\epsilon_{b} V_{b}
$$

and the summed quantity represents the total pore volume. $A_{i}$ this pcint, one could define $a$ volume-averaged porosit:, then write down a source depletion equation stuch as

$$
V_{1}\left\langle\epsilon_{1}\right\rangle_{v}\left[n_{1}^{\prime}(0)-n_{1}^{\prime}(t)\right]=M_{1}^{0}-M_{1}^{*}(t)
$$

The starred value represents the amount of active material remaining at any time, $t$, and the difference in active amounts is the same as that in total amounts. Both differences are equivalent to the cumulative loss $N_{1}(a, t)$ from the source at $i=a$. Notice that diffusion terms and coefficierts are absent.

Some bookkeeping will be required to separate the active and total amounts. First, however, we shall consider changing concentrations to bulk values or pseudo-solid-state values. This must be done eventually since gas transport will not apply to the coating, anc little information is available conceming $\mathrm{p}^{\prime}, \epsilon_{\mathrm{k}}$, and $\epsilon_{\mathrm{b}}$ in the source zone. Consideration of the first equation for the initial quantity in terms of $k$ anc $b$ region is adequate. Under the usual convention, we say that the bulk concentration for region $j$ is $\epsilon_{j} n_{1}^{\prime}(0)$, where $j=$ $k$ or $b$. Then, using mobile or active notation,

$$
V_{k} C_{k}^{*}(0)+V_{b} C_{b}^{*}(0)=M_{i}^{0}
$$

Illustrative histograms depicting initial and bounciary ronditions as visualized for the source region are presented in Fig. 3.

In Figs. 3(a) and 3(b) we show the $k$ and $b$ profiles, with and without contributions of the trapped fraction. One may note that starred and unstarrted bulk concentration (i.e., the active and actual) values are equivalent over $b$, bui not over $k$. Consider the discontinuity at the $k$ - $b$ interface and the concentration ratio

$$
\frac{C_{b}(0)}{C_{k}(0)}=\frac{C_{b}(0)}{C_{k}(0)}=\frac{\epsilon_{b} n_{1}^{\prime}(0)}{\epsilon_{k} n_{1}^{\prime}(0)+\frac{(1-f) M_{1}^{0}}{V_{k}}}=\frac{\epsilon_{b}}{\epsilon_{k}+\frac{(1-f) \Sigma V_{t}}{f V_{k}}}
$$

as it applies to Fig. 3(a). The second term in the denominator accounts for the trapped amount. The viilue 
$\cos 0$ ons $\pi-3027$

(a)

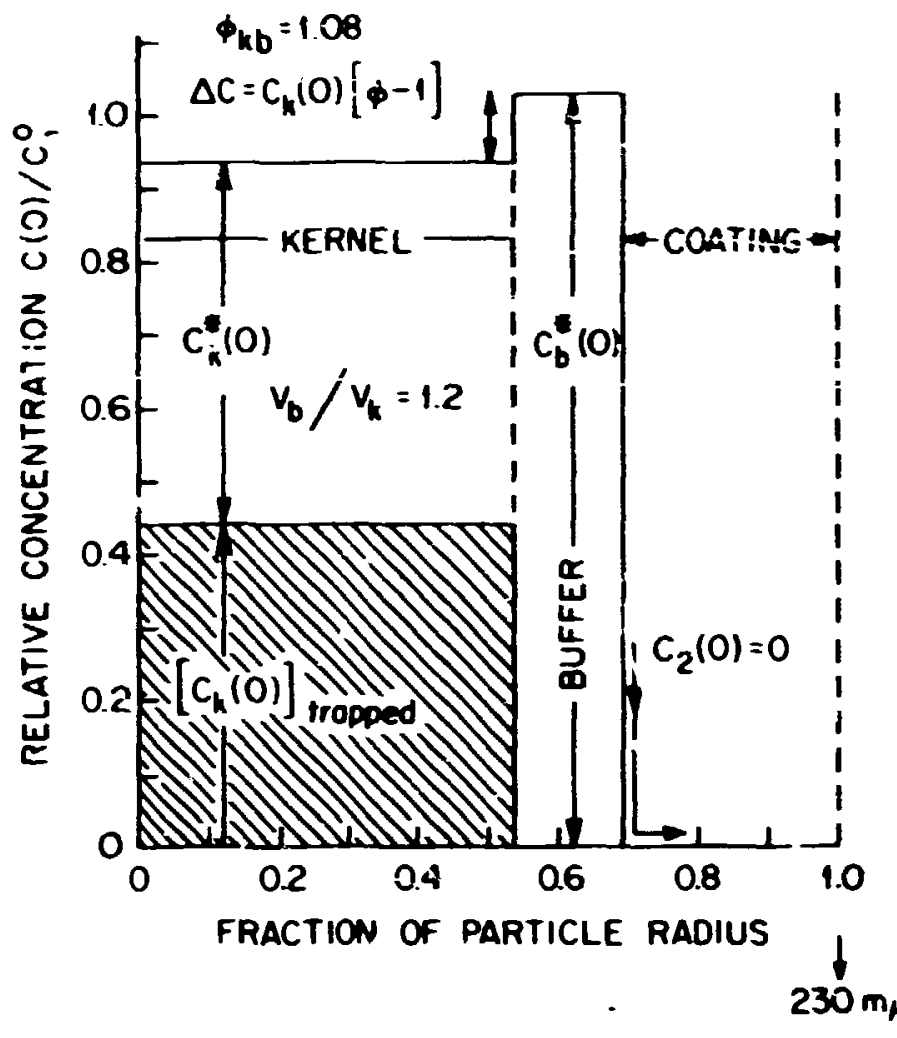

ZONE 1 : KERNEL + BUFFER

ZONE 2: OUTER COATING

(c)

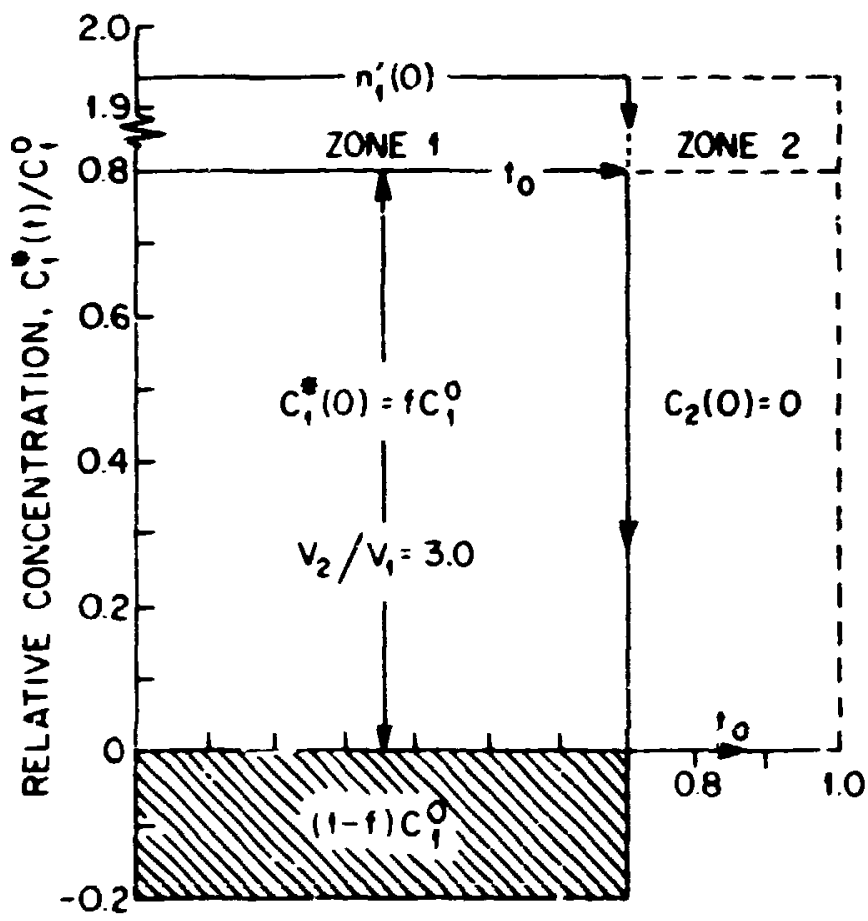

(b)

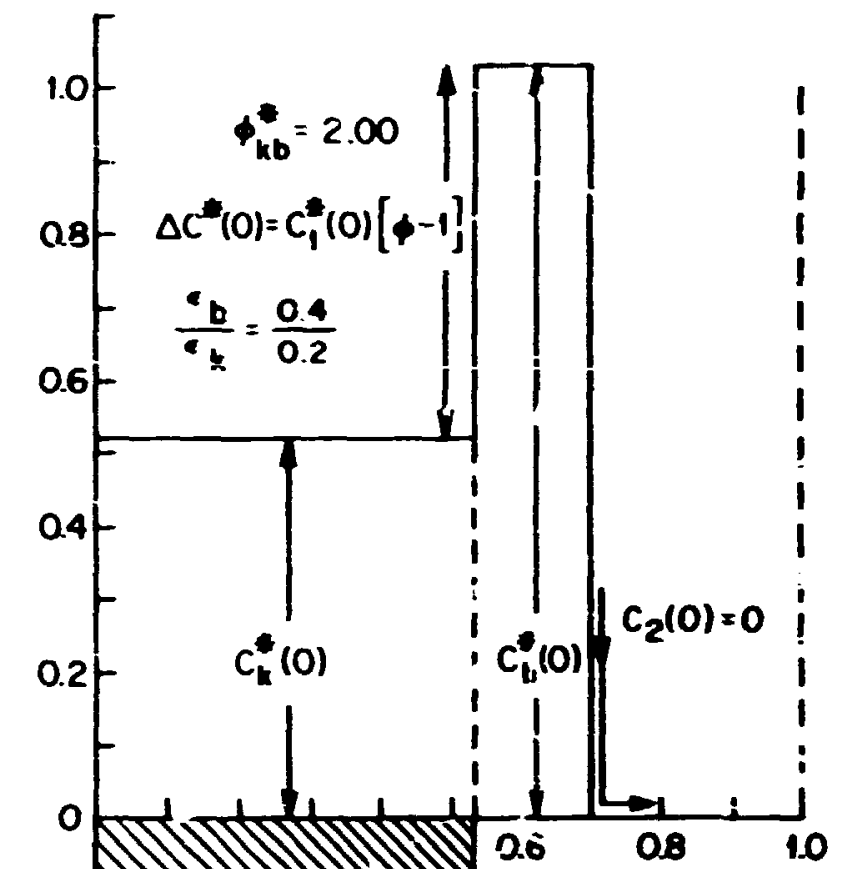

$m_{\mu}$
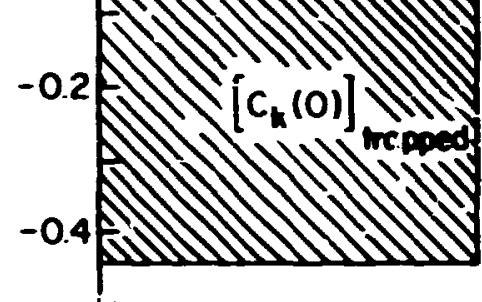

(d)

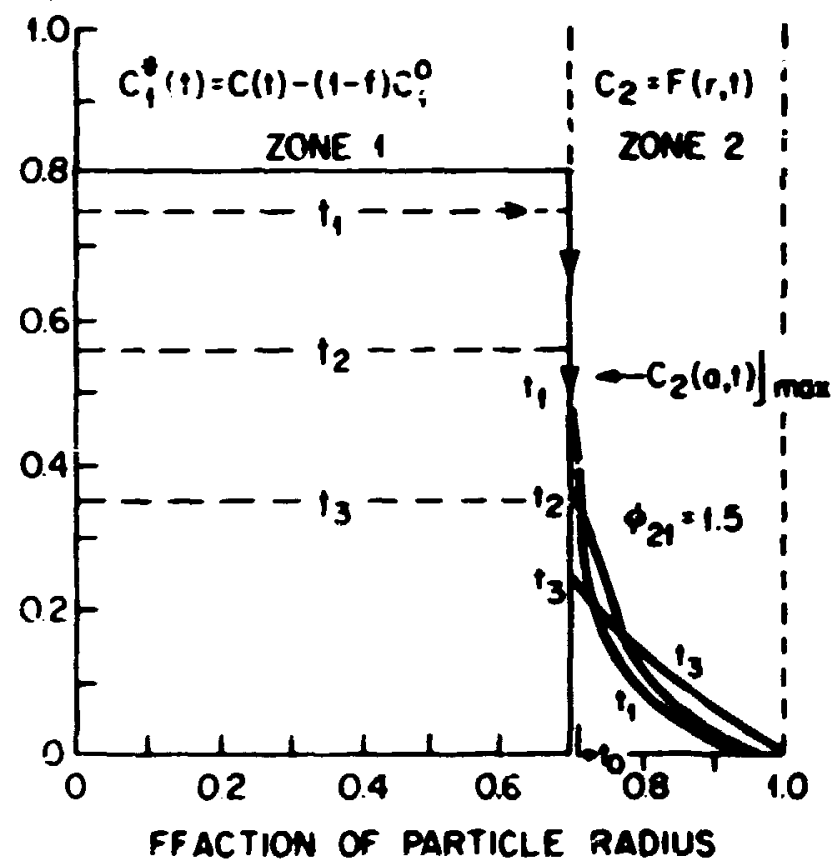

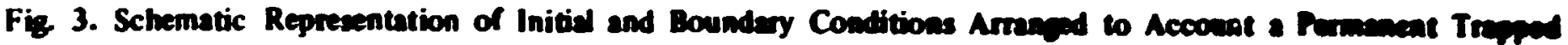
Fraction and Pritition Coefricients. Initial condi!ions are exhibited in (a) through (c): boundary conditions for a depleting sysiem are shown at (d). 


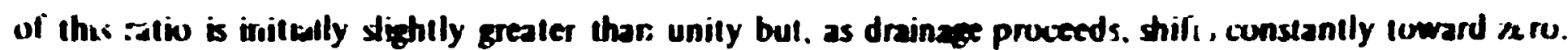
If. on the other hand. the concentration profik is cuns!ra:ted entirely in terms of active concentiations. as in Fig. $\mathbf{3}$ (b). the ratiu becumes meaningful: that is it becumes a time-consant "partition civefficient."

$$
h_{k b}=\frac{C_{b}(0)}{C_{k}(0)}=\frac{C_{b}^{(1)}}{C_{k}(1)}=\frac{\epsilon_{b}}{\epsilon_{k}}
$$

The cuncept of is very simple. almust trite. when applied to the source 4 ne, nevertheless, it has considerable importance when applied to the source-cooting interfase where it refiects gas-solid solubilities.

The general stape of the profites in Fig. $3(b)$ should be retainal throughout th: depletion if $\$$ remains constant. Thus, the shape at anj $t$ is about the same as that $\mathrm{at}=0$ and vice vers. Because there is no reason to arry imu source concentraticns from this point on. a volume average value of the form

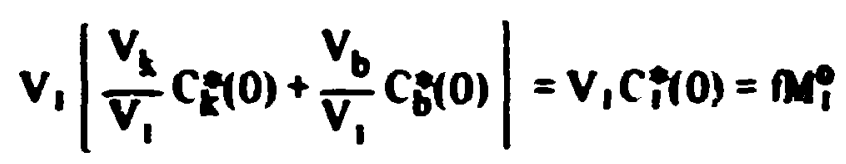

will be used. Consider the concentration relationship:

$$
C_{1}(t)=C_{1}^{*}(t)+\left(1-n C_{1}\right.
$$

where

$$
C_{i}^{*}(0)=\mathrm{fC}_{i}^{0}
$$

With the above. one may write the following differences:

$$
V_{1}\left[C_{1}(0)-C_{1}(t)\right]=V_{1}\left[C_{i}^{0}-C_{i}^{*}(1)\right]
$$

Both are equivalent tc

$$
M_{i}^{0}-M_{1}(1)=M_{i}^{0} \cdot M_{i}^{*}(1)=N_{1}(2,1) \text {. }
$$

After very long periods of tim :. ail differences and $N_{1}($ a. $t \rightarrow \infty) \rightarrow \mathcal{C C}_{1}^{0}$.

The rate of release from th e source zone is

$$
-\frac{d C_{i}^{*}(t)}{d t}=-\frac{\left(\epsilon_{1}\right)}{R T^{\prime}} \frac{d p^{\prime}}{d t}=+\frac{1}{V} \frac{d N_{1}(2, t)}{d t}
$$

where the initial condition is $\mathrm{C}_{\boldsymbol{f}}^{*}(0)=\mathrm{CC}_{\mathrm{P}}$. It is desirable to express cuncentrations as active values in ordet to get a proper connection between zones 1 and 2 via the $\phi_{21}$ as indicated in Fiz. (d).

\subsubsection{Zone 2 - The Pyrocarbon Costing}

Al of the diffusion effects for cesium migration are charged to zone 2. the pyrocarbon cuating. The ouln; boundary at $r=b$ is always assumed to be zero for cesium Iransport. al least for the first round of 
dala correlatikns. tiviry experinental preciaution is taken to ensure that this will be a good approximation: huwerer. in solix in ilances. a hack pressure might exist if (1) the partictes were buried in a matrix with a

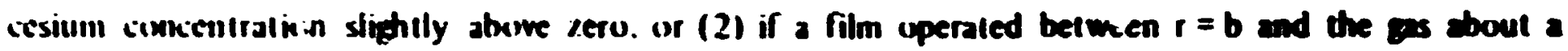
lreestanding parlikte. These effects are must severe for siruntium: comtributions of fidm or evaporation curelficients will be colvsidered laler.

Pyrriartam matrices do nol always have a uniform structure: properties in the initial beposits are

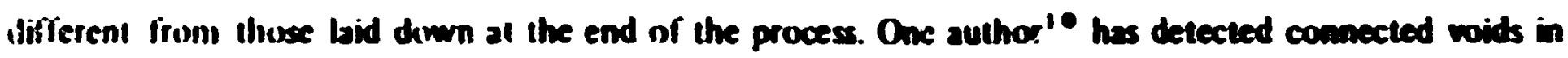
ragions correspunding $: 0 \mathrm{r}=\mathrm{b}$. Furthermore. we have some evidence to show that incressed cesium cincentrations in the craling increase the diffusion coefficients. but only by a factor of 2 or 3.

Aside from the abuve. the region corcesponding $10 \mathrm{r}=\mathrm{a}$ is of $\mathrm{m} \cdot \mathrm{s}$ interest. We consider the ratio

$$
\frac{(i) \cdot 1)}{(2,1)}
$$

and a Henry's law rebalion

$$
n_{1}(1)=K_{c}\left(c_{2}(2.1)\right.
$$

ur

$$
p_{1}^{\prime}(1)=K_{p}\left(c_{2}(2.1)\right. \text {. }
$$

where

$$
R T^{\prime} K_{i}=K_{p} .
$$

The latter stales that ilhere is an equilibrium relationship between the pirtial pressure of the gas and the cincientration in the sulid pyrocarbon. The $C_{2}(a, 1)$ value follows the $p^{\prime}(1)$. up or down. As the pressure is increased. the $K_{p}$ staris 10 increase: then there seems $: 0$ be a limit (a saturation value) as to the amount of cesiun that the pyrocarbun will aciept. If $\mathrm{p}^{\prime}(0)$ exceevs this limit, the $C_{2}(2,1)$ will remain constant until $p^{\prime}(1)$ decreases (1) a lower value where the equilibrium begins to take over again. The ratio will decrease, then assume a cunstarit value such that

$$
\frac{C_{i}^{*}(0.1)}{C_{2}(10.1)}=\frac{\left(\epsilon_{1}\right) m_{1}^{\prime}(1)}{C_{2}(0.1)}=K_{c}\left(\varepsilon_{1}\right)=\phi_{21} .
$$

Since this is the only partition coefficient that will appear in the solutions, the subscript 21 may be eliminated.

Conside: now the regions noted in Fig. 1. particularly regions $A$ and $B$. It is not unreasonable to assurre that pasticles subjected to high burnup levels (>10\% FIMA) will have very high concentrations of unt apped cesium in cone 1 . Thus there may be many instances in which release throughout $A$, and into $B$, is controlled by a constant-potential process. Stated differently, $C_{i}^{f}(a .1)$ will be greater thar the equilibrium o ${ }_{2}$ (a.1) value at the temperature of the experiment such that the coating about $r=a$ is "saturated." and the actual $C_{2}(a .1)$ will be a constant, $C_{2}(a)$. If this happens, a portion of $B$ represents a cunstant release 
rate regime. The unb condition lo be stated at $r=a$ is:

$$
C_{2}(0.1)=C_{2}(a) \text {. }
$$

There is no sune I contribution in the equations. Of cr.urse. zone I depletion cocurs and the rate can be computed, but the value will depend only on interactions in the coatine.

On the other hand. there may be many cases where the release rates are so high (or the burnups are so low) that $C_{2}(a, 1)$ will fall to an equilibrium value at some point in $B$ or $A$. If this or any uther $C_{2}(a, t)$ mode Iransition coccurs in $A$. one would be hard pressed ic .oterpret the data. However, equilibrium attainment in $B$ can be handled. even though it might be difficult to determine the time, $t_{0}$, at which $C_{2}(2,1)$ starts to decrease in ail orderly fashion. Here. one must specify $C_{2}(2,1)$ in terms of $C_{i}^{*}(t)$ p: as well as. a rate equation which gives zone 1 capacity cuntributions to the process. Thus

$$
\boldsymbol{C}_{2}(a, 1)=C_{i}^{*}(1)
$$

and from the last part of the zone I discussion.

$$
\frac{1}{v}\left|\frac{d N_{1}}{d t}\right|_{1 \text { or } 2}=\frac{d C_{f}^{*}(t)}{d t}
$$

or

$$
\frac{1}{V}\left|-A \frac{\partial C_{2}(a, t)}{\partial t}\right|=-\frac{d C_{1}^{*}(t)}{d t}
$$

The initial condition for this rate boundary condition is

$$
C_{i}^{*}\left(i_{0}^{\prime}\right)=f_{i}^{0}
$$

is these derivations. We now turn to the development of diffusion equations, starting with this second set of conditions, for the case where $t_{0}^{\prime}$ corresponds to real time zero. It is convenient to let $A / V=1 / a^{\prime}$.

\section{RELEASE EQUATIONS}

\subsection{Continuous Depletion of Source Potential}

\subsubsection{Radial Flow in Spherical Coordinates}

Consideration is now given to the diffusion phenomena within the coating that govern the fission product retention properties of a fuel particle. We seek a fission product release rate equation, in time-integrated form, that can be ised to prepare a set of "predicted" release curves. Comparisons with experimental curves should permit evaluation of the boundary and initial crnditions used in the derivation and/or suggest the nature of the true conditions. The equations to be derived here represent an extreme limiting case. They will apply only to situations where values of $f$ and/or burnup are relatively low. We shall assume that the highest (the initial) source poteníial will be less than any restrictive hypothetical saturation value. In other words, at time zero, the $\phi$ will "lock in" at a constant value and $C_{2}(a, t)$ will fall in proportion to $C_{i}^{*}(t)$ throughuut the life of the experiment. Transient conditions will prevail at all times. 


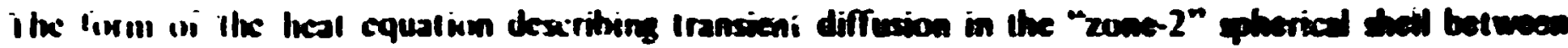
radiil a and h is:

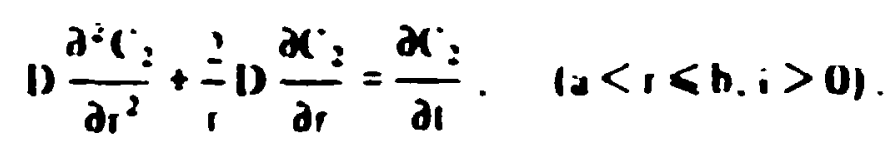

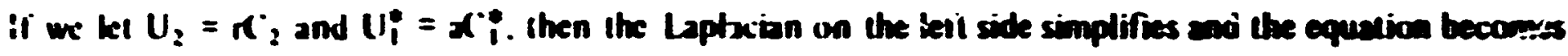
linear in furm: une then obtains

$$
D \frac{\partial^{2} U_{2}}{\partial r^{3}}=\frac{\partial ! !_{2}}{\partial 1}
$$

A i hoike muss ia made here as to the method of approach. The con employ either formal methods or Laplace iransformation teatniques. A formal solution requires a search for a suitable set of property normalized Eigenvatued functions that will converse, in series form, to the rather "sticky" boundiry cunditions associated with this particular probtem. Since this search mingi prove wore diffixult then the original problem. the use of Lapiace transforms seems more reasondble. Thewe are defined by:

$$
\left.f(F(1))=\int_{0}^{-} F(t) e^{s t} d t=R s\right) .
$$

Application of this operation to both sides of the " $U$ " equation gives

$$
D \frac{d^{2} u^{2}}{d x^{2}}=s u_{2} \quad U_{2}(r+0)
$$

The +0 imeans that we cunsider the condition at some $t>0$ and project it back to $t=0$ "from the right." To summarize. we first changed from $C_{2}(r, 1)$ to $U_{2}(r, 1)$ in $r-1$ space. Next, we transformed to $U_{2}(r, s)$ in $r$ s space. where the $r$ contributions can be handled as ordinary differential equations and $t$ contributions are arrived at automatically. in integral form. via inversion back to $U_{2}(r, t)$.

The initial and boundary conditions for Eq. (1) were discusced in detail in the previous section. We inerely enumerate them here.

Iritial conditions:

$$
\begin{aligned}
& C_{l}^{*}(0)=\mathrm{fC}_{1}^{0} . \quad U_{1}^{*}(+0)=\mathrm{afC}_{1}^{0} . \quad(0<r<a) . \\
& C_{2}(r,+0)=0 . \quad U_{2}(r,+0)=0 . \quad(0 \leqslant r \leqslant b) .
\end{aligned}
$$

Boundary cunditions

$$
\begin{aligned}
& C_{2}(b, t)=0 . \quad b u_{2}(b, s)=0 . \quad(t \geq 0) . \\
& \phi C_{2}(a, l)=C_{i}^{*}(a, t) . \quad \phi u_{2}(a, s)=u_{i}^{*}(s), \quad(t>0) .
\end{aligned}
$$


The bas isoundary condition can be troubleavene: it muss be wrilten down carefully:

$$
\frac{\partial C_{1}^{\prime}(1)}{\partial t}=\frac{\mathrm{D}}{\mathrm{a}} \frac{\partial c_{2}(2,1)}{\partial r} . \quad(1>0) .
$$

BuI

$$
\mathbf{U}=\mathbf{r} \text {. }
$$

and

$$
\frac{\partial C_{2}}{\partial r}=\frac{1}{r} \frac{\partial U_{2}}{\partial r} \frac{U_{2}}{r}
$$

Thus

$$
\frac{\partial U_{i}^{*}(t)}{\partial(t)}=\frac{D}{a}\left|\frac{\partial U_{2}(2 . t)}{\partial t}-\frac{U_{2}(2, t)}{a}\right| .
$$

The transformed version is:

$$
\operatorname{su}_{i}^{*}(s) \quad U_{i}^{*}(+0)=\frac{D}{a^{\prime}}\left|\frac{d u_{2}(a, s)}{d r} \cdot \frac{u_{2}(a, s)}{a}\right| .
$$

We recall here that a' represents V/A. Now this boundary condition can be alicied using IC(2) and $\mathrm{BC}(4)$ as follows:

$$
\phi \mathrm{su}_{2}(\mathrm{a} . \mathrm{s})-\mathrm{afC}_{1}^{0}=\frac{D}{\mathrm{a}}\left|\frac{d \mathrm{u}_{2}(\mathrm{a}, \mathrm{s})}{\mathrm{dr}} \ldots \frac{\mathrm{u}_{2}(\mathrm{a}, \mathrm{s})}{\mathrm{a}}\right|
$$

It tums out that the second term in the brackets introduces an inordinate anount of algebraic difficulty in the inversion procedures.

The next step is to solve Eq. (1) in light of IC(3). We have

$$
\frac{d^{2} u_{2}}{d x^{2}}-\frac{s}{D} u_{2}=0
$$

a solution is

$$
u_{2}=B_{1}(s) e^{+r(s / D) 1 / 2}+B_{2}(s) e^{-r(s / D) 1 / 2} .
$$

To satisfy $\mathrm{BC}(4)$ and accommodate boundary corditions at $\mathrm{r}=\mathrm{a}$, we let

$$
B_{1}(s)=\frac{A(s)}{2} e^{b(s / D) i / 2}
$$


and

$$
\left.B_{2}(s)=+\frac{A(s)}{2} c+b(s) b\right) 1 / 2
$$

Tiken

$$
u_{2}=A(s) \sinh \mid\left(b \quad\left|x s^{\prime} D\right|^{1 / 2} \mid .\right.
$$

Ind

$$
\frac{d u_{2}}{d r}=A(s)\left\{(s / D)^{1 / 2} \cosh \left\{(b \quad r M s / D)^{1 / 2} \mid\right\}\right.
$$

When $l=$ a. $b \quad t$ becomes $l=b$ a. Now information f:om Eq. $\left(\pi_{b}\right)$ can be inserted into Eq. (6a) to yield an expression for $A(s)$. which can then be applied in Eq. (T). The result is:

$$
u_{2}(x s)=\frac{\operatorname{s}^{\prime} k C_{1}^{0} \sinh \left|(b-r)(s / D)^{1 / 2}\right|}{\left(s+\frac{D k}{a^{2}}\right) \sinh \left[(a / s / D)^{1 / 2}\right]+\frac{k}{a}(s / D)^{1 / 2} \cosh \left[q(s / D)^{1 / 2}\right]},
$$

where $k=a i \mathbf{a}^{\prime} \phi$.

We now have the solution for $U_{2}(r, s)$ in $r$ - s space. An inversion formula is required for conversion to I I space. It is doubiful that the appropriate $F(t)-v s-f(s)$ relationship has been tabulated for $v_{2}(r, s)$. The only recuurse is to use the inversion integal of the product $e^{\lambda 1} f(\lambda)$, where $\lambda$ is the dummy variable of integration for $s$ expressed as a complex variable and $f(\lambda)$ represents $u_{2}(x, s)$. The result we seek is an

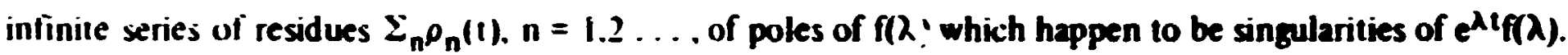
These ikicur at poies of $u_{2}(r s)$ pusitioned at $s_{n}$ values that tend to make $u_{2}(r, s) \rightarrow \infty$.

First. we examine $u_{2}(x, s)$ to determine if it has a siblgularity at $s=0$. This is done by expanding the hyperbolic functions in :erms of series and taking the limit as $s \rightarrow 0$; because the limit has a finite value, there is no singularity at $\mathrm{s}=\mathbf{0}$ for $\mathrm{Eq} .(9 \mathrm{c})$. If a singularity were found, we would attempt to remove it by forming the product (s - 0$)_{2}(x . s)$. Successful removal makes the $s=0$ singular point a simple pole. The limit of the product. as $s \rightarrow 0$. wouk be the residue of the pole, and also part of our inverted solutions.

Nonzero singularities are found by setting the denominator of Eq. (7a) equal to zero, but first let us write:

$$
u_{2}(x . s)=\frac{p(s)}{q(s)}
$$

and

$$
Q(s ; D)^{1 ; 2}=i \alpha
$$

One finds that

$$
p(s)=i\left(a^{\prime} k f C_{1}^{0}\right) \sin [(\alpha / \ell)(b-r)]
$$


ind

$$
q(s)=i l)\left\{\left|\frac{a^{2}}{l^{2}}+\frac{k}{a^{2}}\right| \sin a+\left|\frac{a k}{a l}\right| \cos a\right\} .
$$

The in:aginary number i will cancel in the ratio, and $q(s)$ will be zero when

$$
\cot a_{n}=\frac{2 a_{n}}{9 k}-\frac{l}{2 a_{n}}=\frac{R a_{n}}{k} \cdot \frac{1}{R a_{n}} .
$$

Intersection of the cota..gent curves and a hyperbola locates the $a_{n}$ in each $(0, \pi)+\ldots+(n \pi)+(n+1) \pi$ interval. At high $n$, the values approach $n \pi$. Although there is an equivalent set of negative $\alpha_{n}$ 's, v,e can only use the positive ones [see Eq. (7)]. There are no repeated $\alpha_{n}$ values, and all are real. Equation $(8)$ is called the auxiliary equation, and the $a_{n}$ 's are found to be the roots of o.it final equations. Since

$$
s_{n}=-\frac{a_{n}^{2} D}{l^{2}} . \quad n=1,2,3, \ldots
$$

all poles will lie on the negative real axis in the complex s plane. The poles are "simple." In this case, the problem of inversion reduces to the application of:

$$
F(t)=\sum_{l}^{\infty} \frac{p\left(s_{n}\right)}{\left[d q / d s ! s_{n}\right.} e^{+s_{n} t}
$$

Equation (II) gives a simple recipe for obtaining $U_{2}(r, t)$. We find $d q / d a$, then $d q / d s$, and form the series indicated:

$$
\begin{aligned}
& \frac{d q}{d \alpha}=-\frac{D}{l}\left[\left(\frac{a \alpha}{l}-\frac{k \alpha}{a}\right) \sin \alpha \cdot\left(\frac{\alpha^{2}}{l}-\frac{k l}{a^{2}}-\frac{k}{a}\right) \cos \alpha\right], \\
& \frac{d q}{d s}=\left(\frac{d \alpha}{d s}\right)\left(\frac{d q}{d \alpha}\right)=-\left(\frac{l^{2}}{2 D a}\right)\left(\frac{d q}{d \alpha}\right), \\
& \left.\frac{d q}{d s}\right]_{\alpha_{n}}=\frac{l}{2}\left[\left(\frac{2}{l}+\frac{k}{a}\right)+\left(\frac{\alpha_{n}}{l}-\frac{k l}{\alpha_{n} a^{2}}-\frac{k}{\alpha_{n} a}\right)\left(\frac{a}{k}\right)\left(\frac{\alpha_{n}}{l}-\frac{k l}{\alpha_{n} a^{2}}\right)\right] \sin \alpha_{n} .
\end{aligned}
$$

The auxiliary equation, Eq. (9), was used to factor out the $\sin \alpha_{n}$; for this reason, the subscript $n$ appears on the $a$ 's. Now let $\alpha_{n}=\alpha_{n}^{\prime} \ell$. This is done because we seek a specific form for $(\mathrm{dq} / \mathrm{ds}) \alpha_{n}$ which will allow comparisons with other related solutions cataloged by Carslaw and Jaeger. " From these we can write an identity required in the present problem. Continued manipulations yield:

$$
\left.\frac{d q}{d s}\right]_{\alpha_{n}^{\prime}}=\frac{a}{2 k}\left[\left(\frac{k}{a}+\frac{k^{2} \ell}{a^{2}}+\frac{k^{2}}{a^{3} \alpha_{n}^{\prime 2}}\right)+\ell\left(\alpha_{n}^{\prime}-\frac{k}{a^{2} \alpha_{n}^{\prime}}\right)^{2}\right] \sin \alpha_{n}^{\prime} \ell
$$


The final rusult is obtained when one seeks ou! the various factors assuciated with

$$
\frac{k^{2}}{a^{2}} \cot ^{2}\left(a_{n}^{\prime} \psi\right)
$$

and

$$
\left(2 \frac{k}{a} \frac{k^{2}}{a^{2} a_{n}^{\prime 2}}\right) \cot \left(a_{n}^{\prime} l\right)
$$

Thus.

$$
\left.\frac{d q}{d s}\right]_{\alpha_{n}}=\frac{2 k\left(a_{n}^{\prime} l\right)+4\left(a_{n}^{\prime} a\right) \sin ^{2}\left(a_{n}^{\prime} l\right) k \sin \left(2 a_{n}^{\prime} l\right)}{4\left(a_{n}^{\prime} a\right) \sin \left(\alpha_{n}^{\prime} l\right)} .
$$

We define $Q\left(a, k, a_{n}^{\prime}, l\right)$ as the numerator of this expression.

A combination of Eqs. (8), (9), and (12), according to Eq. (11), completes the inversion:

$$
U_{2}(r, t)=\sum_{1}^{\infty} \frac{4 a a_{n}^{\prime}\left(a^{\prime} k f C_{1}^{0}\right) \sin \left(a_{n}^{\prime} l\right) \sin \left[x_{n}^{\prime}(b-r)\right]}{Q\left(a, k, a_{n}^{\prime} l\right)} e^{-D a_{n}^{\prime} t}
$$

where $U_{2}(r, t)=r C_{2}(r, t)$.

Expressions for the flux are:

$$
J(r, t)=-D \frac{\partial C_{2}(r, t)}{\partial r}=-\frac{D}{r} \frac{\partial U_{2}(r, t)}{\partial r}+\frac{D}{r^{2}} U_{2}(r, t)
$$

At $r=b$. the last term on the right is zero, and the flux out of the particle is every term in Eq. (13) multiplied by the factor

$$
\left(-D K-a_{n}^{\prime} / b\right) \cos \left[\alpha_{n}^{\prime}(b-b)\right\}=D \alpha_{11}^{\prime} / b
$$

The time-integrated flux is:

$$
j_{0}^{i} J(b . l) d t=\sum_{l}^{\infty} \frac{4 a\left(a^{\prime} k f C_{1}^{0}\right) \sin \left(c_{n}^{\prime} \ell\right)}{b\left[Q\left(a, k, \alpha_{n}^{\prime} \ell\right)\right]} \int_{c}^{i} e^{-D \alpha_{n}^{\prime 2} t} d\left(D \alpha_{n}^{\prime 2} t\right)
$$

and the integral is $: 1 \cdot e^{-D \alpha_{n}^{\prime 2} t^{\prime}}$.

Consider now the cumulative fractional release written as:

$$
F R=\frac{N(b, i)}{M_{1}^{0}}=\frac{N(b, \bar{t})}{V_{1} C_{i}^{0}}
$$


where

$$
N(b . i)=4 \pi b^{2} \int_{0}^{i} J(b . t) d t
$$

If one recalls that $a^{\prime}=V_{1} / 4 \pi a^{2}$, the following equation can be obtained by appropriate substitutions and manipulati:airs:

$$
\frac{F R}{i}=\frac{4 k b}{a} \sum_{1}^{\infty} \frac{\left(1 \quad e^{-D a_{n}^{\prime} i}\right) \sin \left(\alpha_{n}^{\prime} \ell\right)}{Q\left(a \cdot k \cdot \alpha_{n}^{\prime} l\right)}
$$

Eq. (14a) ian be expressed as twc: series: one without, and one with, an exponential term. With the aid of another solution given by Carslaw and Jaeger, ${ }^{12}$ it can be shown that the first series cc respondi! $a \bar{b}$ iv $t=0$ converges to unity. Thus, with $\alpha_{n}=\alpha_{n}^{\prime} l$.

$$
\frac{F R}{f}=1-\frac{4 k b}{a} \sum_{l n}^{\infty} \frac{e^{-\alpha_{n}^{2} T} \sin \alpha_{n}}{\left[2 k a_{n}+\left(4 a a_{n} / l\right) \sin ^{2} \alpha_{n}-k \sin 2 \alpha_{n}\right]},
$$

where

$$
T=\frac{D i}{(b-a)^{2}}, \quad k=\frac{a}{a^{\prime} \phi}=\frac{3}{\phi} .
$$

and $x_{n}$ are the positive roots of

$$
\cot \alpha_{n}=\frac{a \alpha_{n}}{k(b-i)} \quad \frac{(b-a)}{a \alpha_{n}}: n=i .2,3 \ldots
$$

\subsubsection{Linear Flow in Slab Geometry}

In view of the complexities of Eq. (14b), we explore the possibility of approximating radial flow with linear flow eqiations. The same initial and boundary conditions tilat were used previously will be applied here. The distinguishing feature is that we will preiend that $A(r)$ can be represented by a position constant average of $4 \pi \mathrm{ab}$. Tilen the " $\mathrm{U}^{\cdot}$ substitution can be eliminated, and one may write

$$
D \frac{\partial^{2} C_{2}}{\partial x^{2}}=\frac{\partial C_{2}}{\partial t}
$$

Initial conditions:

$$
C_{1}^{*}(0)=f C_{1}^{0} . \quad C_{2}(x+0)=0
$$

Boundary conditions:

$$
C_{2}(b, t)=0, \quad \phi C_{2}(a, t)=C_{1}^{*}(a, t)
$$


alld

$$
\frac{\partial C_{i}^{*}(1)}{\partial t}=\frac{D A}{V} \frac{\partial C_{2}(1,1)}{\partial x}
$$

The procedure is exactly the same as outlined in Sect. 3.1.1. One obtains the transformed equation:

$$
c_{2}(x . s)=\frac{i f C_{1}^{0} \sin |\alpha / l(b \quad x)|}{\frac{i \phi D}{l}\left(\frac{\alpha^{2}}{l} \sin \alpha+h \cos \alpha\right)}
$$

where $!=\leq /$ VQ

The inverse is:

$$
C_{2}(x, t)=\sum_{i}^{\infty} \frac{2 h f C_{1}^{0} e^{-D \alpha_{n}^{\prime 2} t} \sin \left[\alpha_{n}^{\prime}(b-x)\right]}{\phi\left[l\left(\alpha_{n}^{\prime 2}+h^{2}\right)+h\right] \sin \left(\alpha_{n}^{\prime} l\right)}
$$

A form of this kind is given by Carslaw and Jaeger. ${ }^{13}$ Converting to the flux and then integrating with respect to time gives:

$$
\frac{F R}{i}=1 \quad \sum_{1}^{\infty} \frac{2 h e^{-1) \alpha_{n}^{\prime} i}}{\left[l\left(a_{n}^{\prime 2}+h^{2}\right)+h\right] \cos \left(\alpha_{n}^{\prime} l\right)}
$$

Let $a_{n}=a_{n}^{\prime} \ell . L=\ell l$. and $T=D \tilde{t} / \ell^{2}$. Then Eq. (15) evaluated at $x=b$ can be written as:

$$
\frac{C_{1}^{*}(a . t)}{C_{i}^{*}(0)}=\sum_{1}^{\infty} \frac{2 L e^{-\alpha_{n}^{2} T}}{\left[L(L+1)+\alpha_{n}^{2}\right]}=1-f_{1}(L . T)
$$

and

$$
\frac{F R}{i}=1 \quad \sum_{1}^{\infty} \frac{2 \mathrm{U}\left(\sec \alpha_{n}\right) e^{-\alpha_{n}^{2} T}}{\left[\mathrm{~L}(\mathrm{~L}+1)+\alpha_{n}^{2}\right]}=r_{2}(L, T)
$$

The $\alpha_{n}$ here are roots of:

$$
\alpha_{n} \tan \alpha_{n}=\text { L. } n=1,2,3, \ldots
$$

A detailed ap-ivation of Eq. ( $16 \mathrm{~b})$ is given by Raines et al. ${ }^{5}$ The $f(L, T)$ functions are discussed by Jaeger and Ciarke ${ }^{4}$ and Carslaw and Jaeger." 


\subsubsection{Discussion}

Inspection of the release relationships Eys. (14b) and (16b) reveals that very little can ie done regarding correlation of depletion data in the absence of an $f$ value. All apparen: fractional release values must be

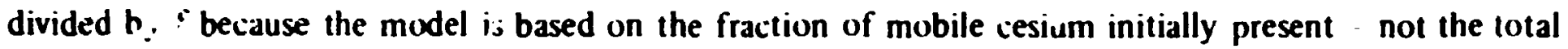
value. Ti.is adjustment tends to :teepen the original curve in such a way that ter.ninal values will approach unity in complian ce with the $T \rightarrow \infty$ limit of the derived release functions. In many cases, the problem relates to conseristion of experimental time: it is not feasible to run experiments for long times just to obtain $\mathrm{f}$. The best approach for obtaining an accelerated determination woulu be to crack the particle. remove the coating. and anneal the remaining componients at the temperacure of interest. However, it may be possible to estimate $t$ if the curves account for about half the mobile amount. $O^{f}$ course, this procedure is somewhat complicated because one must coupie this estimate with estimates for $\mathrm{L}$ or $\mathrm{k}$ and $\mathrm{D}$.

Pi.us of the two functions under discussion are shown in Fig. 4. For sample calculations covering the spherical case. it was necessary to select a particular $\mathbf{R}$ representing an average collection of particles. Considerations of a different collection would require another $R$ value and computations of a complete new set of curves giving FR is a funition of $D t / l^{2}$ at various $k$ values. In the linear flow case, one set of curves is adequate. The objective of presenting the plots in Fig. 4 was to provide some indication as to the shapes of the curves on an FR-vs-log $T$ basis, and aiso to demonstrate how effectively spherical results can be approximated with linear results. It is only necessary to select a set of $L$ values which will correspond to the various $k$ values. If we take $A=4 \pi$ ab in the expression for $L$, then

$$
L=\frac{l A}{V_{\phi}}=\frac{(b-a)(4 \pi a b)}{\phi(4 / 3) \pi a^{3}}=\frac{3(b-a) b}{\phi a^{2}}=\frac{b(b-a) k}{a^{2}}
$$

where $\phi=3 / \mathrm{k}$.

What we have actially done is 10 "smear" the source volume out over an average area to define an effective source depith, and then to postulate that $\phi$ is the same in each system. Of course, if we start discussing concentration profiles. the problem should be approached in another way. The above resuit is adequate. however, for a rough correlation of release data. Figure 5 shows a plot of the curves in rectangular coordinates. Figure 6 gives a plot of Eq. (15), demonstrating the high initial source zone depletion rates. For example. at $L=1.0$ and $T=0.4$, the source zone has lost $46 \%$ of its initial active concentration [Fig. 6] the corrected release at $r=b$ is only $16 \%$ [Fig. 5].

Brief descriptions of the curve fitting and parameter extraction procedures are in order. We assume that the final measured cumulative release is high enough to permit at least a "ball park" estimate of $f$. If an e;timate of maximum saturation con:entration of the fission product in the coating of interest is also available. the constant-potentiai correlations (given in the next section) should be employed to ensure that this value was not exceeded during the experiment being anaiyzed. With the assurance that constantpotential bet avior did not intrude into the resulss. one may correct the data to give $F R / f$ values and plot these as a function of $\log$ [real time. : $]$. The experimental semilog plots can $b$ ' 'ined up with theoretical semilog plots [see Fig. 4 - dotted curves] by parallel translations cf the $t$ and $T$ axes. At this point. tentative values of $f, D$, and $L$ may be selected. We assume that the data have already been plotted on rectangular paper and that an arage slope. $m$, and $t_{0}$ have teen determined. From the curves in Fig. 5 . one may obtain a $T_{0}-v s-L^{-1}$ curve and an $m^{\prime}$-(theoretical slopes)-vs- $L^{+1}$ curve. All this information, along with the relationship

$$
m=\left(D f / l^{2}\right) m^{\prime}
$$




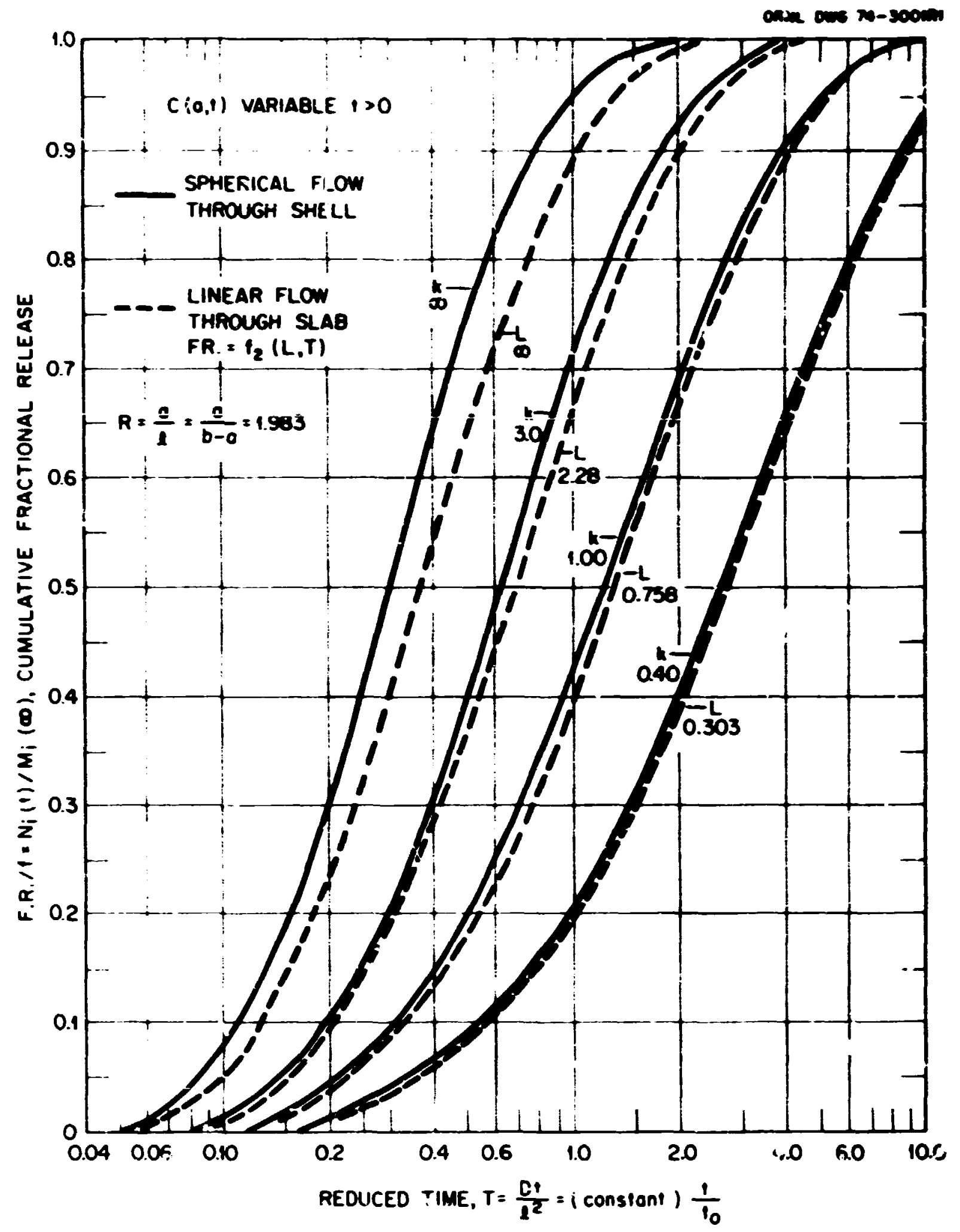

Fig. 4. Depletion rurves Predicted on the Bass of kquations (14b) and (16b). (urves show the relatiosships between FR through the coating). T, and $k$ or $L$ (actually $\phi)$ for spherical and linear flow gecmetries when the cesi.m concentration in the coating nearest the source decreases continuously uith time.

can be juggled aoout to establish final values. Later, these can be checked against the orizinal semilog plots.

To summarize. we need to pvaluate three parameters or unknowns, and unless we have three pieces of curve informa'ion that are equivalent to three equations. this procedure will degenerate to a bootstrap engineering exercise. The most effectiv: ap rroach is to measure $f$ separately. We now consider cases wherc release does not depend on $f$ and either $\phi$ or $L$ values - in other vords, cases where the source potential is so higf, that the coating nexc to li:e cource becomes saturated and maintains a time-constant concentration. 


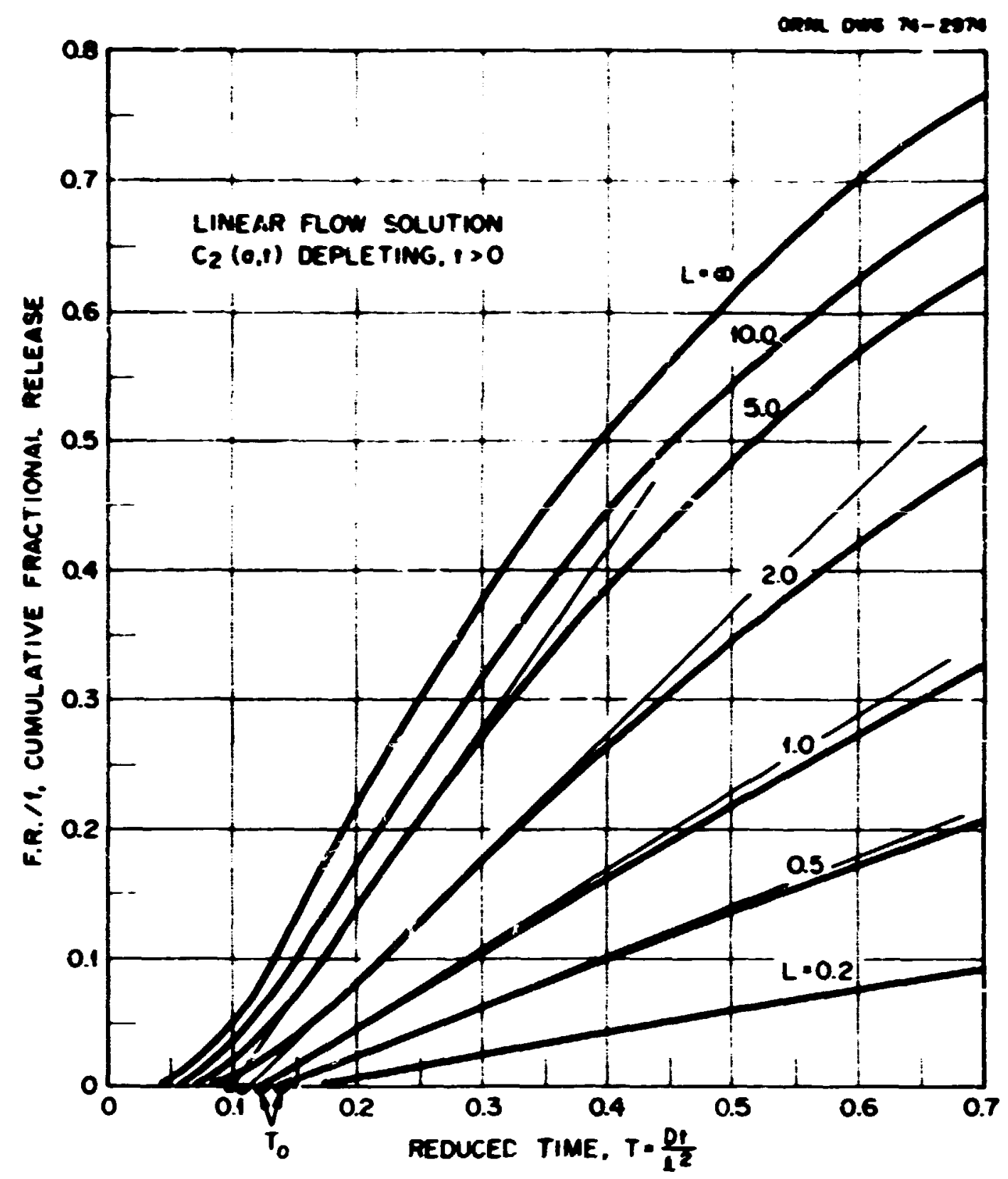

Fia. 5. Shapes of the Linear Depletion Curves of Fiare 4 When Plotted on Rectangular Cocordinates. Each constant $L$ curve has a different $T_{0}$ inter:ept. which means that a different constant must be used to evaluate $D$ in terms of breakthrough time. $t_{0}$.

\subsection{Constant Potential at the Source Boundary}

\subsubsection{The Complete Solution}

A rather general approach will bz employed here in that the equations will be developed using the assumption that an external mass :ransfer film. or an evaporation coefficient, is operating at the outer boundary at $r=b$. The possible influence of film effects cannot be ignored. We have collected limited data which suggest that films are of importance in strontium transport, and we have no evidence that they are absent in CRNL cesium release experiments. ${ }^{*}$ It develops that the effects of film resistances, actually admittances. cannot be detected in the results from a typical release experiment, even in the initial transient regime.

\footnotetext{
* Note added in proof: Actually, we are not concerned here with films or evaporation coefficients associated with high-pressure laminar flow or low-pressure Knudsen flow, per se. Rather, we anticipate that contributions of sacrificial layers or partition coefficients at $\mathrm{r}=\mathrm{b}$ might be treated in terms of a film analog.
} 


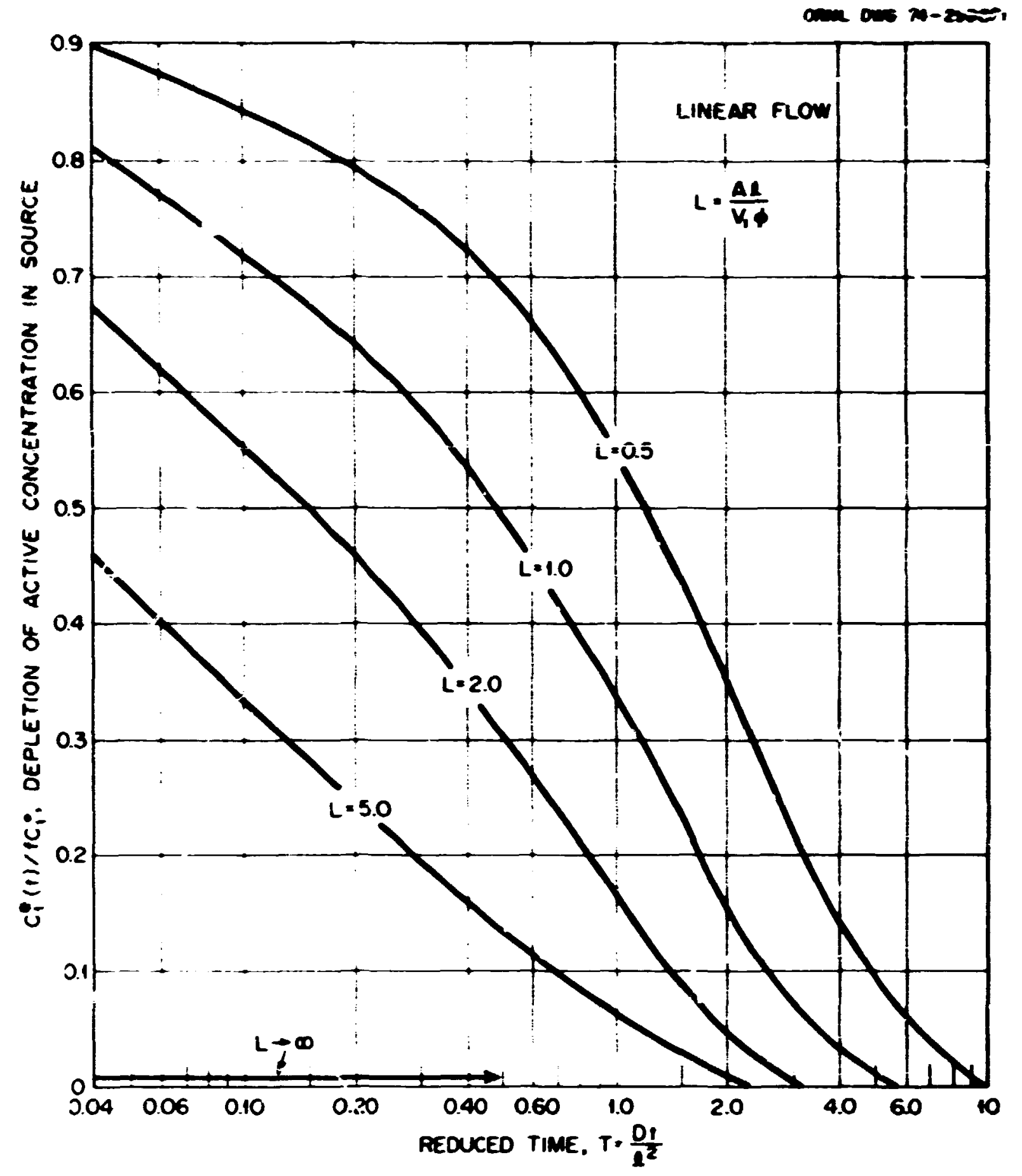

rig. E. Depletion of the Source Region When Equation (16b) Dexcribes Overall Prticle Redeare.

Again we shall adopt equations, as described by Jaeger and Clarke. ${ }^{4}$ in linear flow coordinates. These will be adequate for present purposes. It should be noted that the solutions for the liniting case where $L^{\prime}=\mathrm{lh}^{\prime} / \mathrm{D} \rightarrow \infty$. $\mathrm{h}^{\prime}$ being the film coefficient, are identical for both spherical and linear systems. The only difference is that one must employ $A=4 \pi a b$ in the spherical case.

Two assumptions are made: (1) that the potential ai $r=a$ will remain constant long enough to establish a reasonable period of steady-state release. and (2) that the coating did not take up cesium during irradiation. Thus, we have the initial condition

$$
C_{2}(x, 0)=0 . \quad a<x \leqslant b .
$$

?ith the following soundary conditions:

$$
C_{2}(a, t)=C_{0}, \quad t>0 ; \quad c_{2}(a, s)=C_{0} / s .
$$


and

$$
D A \frac{\partial C_{2}(b .1)}{\partial x}=h^{\prime} A\left|C_{2}(b .1) \quad 0\right|
$$

which can aiso be written in transformed $x$ s space as

$$
\frac{d i_{2}(x, s)}{d x}=-H v_{2}(b . s)
$$

and

$$
H=\frac{h^{\prime}}{D}
$$

These conditions apply to:

$$
D \frac{\partial^{2} C_{2}(x, t)}{\partial x^{2}}=\frac{\partial C_{2}(x, t)}{\partial t}
$$

or

$$
s c_{2}(x . s)-C_{2}(x,+0)=D \frac{d^{2} c_{2}(x . s)}{d x^{2}}
$$

We now m.jve the $\mathrm{c}$ :-gin to position $\mathrm{r}=\mathrm{a}$, let $\mathrm{b}=\ell$, and eliminate the subscripr 2 . A solution to Eq. (21) that satisfies all conditions is:

$$
c(x . s)=\frac{C_{0}}{s}-\frac{C_{0} H \sinh x(s / D)^{1 / 2}}{s\left[(s / D)^{1 / 2} \cosh f(s / D)^{1 / 2}+H \sinh \ell(s / D)^{1 / 2}\right]}
$$

There are three parts to the solution: the inverse of the first term, $C_{0}$; $s \rightarrow C_{0}$; the residue of the simple pole at $s=0$ from the second term, which we call $\zeta(s, x)$; and a series of residues from $\zeta(x, s)$ for nonzero singularities. The $s=0$ residue is:

$$
\operatorname{Lim}_{s \rightarrow 0} s \zeta(x, s)=-\frac{C_{0} H x}{1+H \ell}
$$

The first two parts give:

$$
C_{0}\left[1-\frac{H x}{1+H \ell}\right]=C_{0}\left[\frac{1+L^{\prime}(1-x / l)}{1+L^{\prime}}\right]
$$

this is the steady-state solution with $L^{\prime}=\ell H$. Only $s=0$ contributions of $\zeta(x, s)$ have been accounted for; the remainder of the problem is to take the inverse of $\zeta(x, s)$ (with the $1 /$ s still in). If we let 


$$
(4: D)^{1 / 2}=8
$$

one eventually finds that

$$
C(x, 1)=C_{0}\left[\frac{1+L^{\prime}(1 x / R)}{1+L^{\prime}}\right] \sum_{!}^{-} \frac{2\left(\beta_{n}^{2}+L^{\prime 2}\right)^{-\beta_{n}^{2} T} \sin \left(\beta_{n} x / \ell\right)}{\beta_{n}\left[L^{\prime}+L^{\prime 2}+\beta_{n}^{2}\right]}
$$

with an auxiliary equation tinat gives the $\beta_{n}$ pusitive roots, $n=1,2,3, \ldots$, as follows:

$$
\beta_{n} \cot \beta_{n}+L^{\prime}=0
$$

with

$$
\mathbf{L}^{\prime}=\mathbf{Q H}=\mathbf{\ell} \mathbf{h}^{\prime} ! \mathbf{D} .
$$

\subsubsection{General Relesse Equation}

The resuli obtained by Jaeger and Clarke, ${ }^{19}$ when the above is manipulated to form the flux at $x=2$ and integrated to ohtain the cumulative release, was:

$$
\frac{N_{1}(b, t)}{C_{0} A l}=\frac{L^{\prime} T}{1+L^{\prime}} \cdot \frac{L^{\prime}\left(L^{\prime}+3\right)}{6\left(I^{\prime}+1\right)^{2}} \cdot\left[1+\sum_{1}^{\infty} \frac{(12) L^{\prime}\left(L^{\prime}+1\right)^{2} e^{-\beta_{n}^{2} T} \sec \beta_{n}}{\left(L^{\prime}+3\right)\left[L^{\prime}\left(L^{\prime}+1\right)+\beta_{n}^{2}\right]}\right] .
$$

Everything inside the bracket was defined simply as $\varphi_{4}\left(L^{\prime}, T\right)$. The latter approaches unity within a reasunable fraction of experimental diffusion $i$. or theoreticis reduced time, $T$ (see Fig. 7). Thus the vifference of the second ter. $n$ in Eq. (23c) vanishes. and one obiains:

$$
\Delta\left[N_{1}(b, t)\right]=A \ell\left[\frac{L^{\prime} \Delta T}{l+L^{\prime}}\right] C_{0}=\frac{(A \ell)\left(l h^{\prime} / D\right)\left(D \Delta: / \ell^{2}\right) C_{0}}{\left(1+\ell h^{\prime} / D\right)},
$$

and

$$
\frac{\Delta N_{1}(b, t)}{\Delta l}=A C_{0}\left[\frac{1}{h^{\prime}}+\frac{l}{D}\right]^{-1}
$$

This is the steady-stat $\epsilon$ flow equation with both film and diffusion coefficients contributing. If everything but $C_{0}$ is known. and

$$
\frac{\Delta N_{1}(b . t)}{\Delta t}=M_{1}^{0} \frac{\Delta(F R)}{\Delta t}=M_{1}^{0} m
$$

where $m$ is the slope of the tractional release curve, then

$$
C_{0}=\frac{M_{1}^{0} m}{A}\left[\frac{i}{h^{\prime}}-\frac{\ell}{D}\right]=\frac{M_{1}^{0} m}{A l}\left[\frac{1+L^{\prime}}{L^{\prime}}\right] \frac{l^{2}}{D} \text {. }
$$




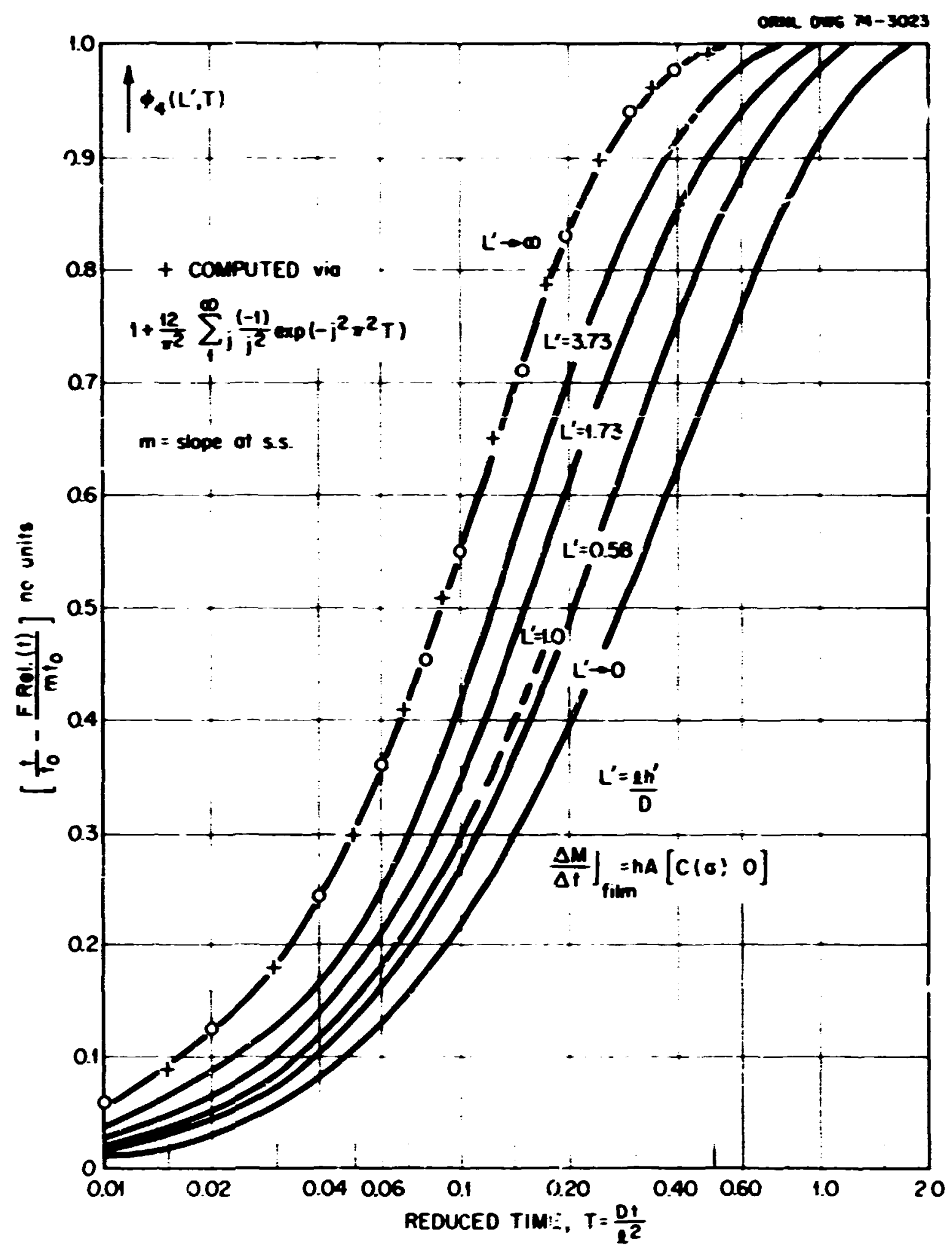

Fig 7. Functions That Govern Approsch to Sisdy-State Constant-Potential Release. These transient curves show release as a function of reduced time for various reduced ratios of the film coefficient to diffusion coefficient.

We shall now temporarily depart from this discussion to consider extrapolation of the $y=N_{1}(b . t) / C_{0} A l$ f:ncticn, at $T$ values corresponding to steady-state operation, back to $y=0$ with $\varphi_{4}\left(L^{\prime}, T\right)=1$. Thus

$$
\frac{L^{\prime} I_{0}}{I+L^{\prime}}=\frac{L^{\prime}\left(L^{\prime}+3\right)}{6\left(L^{\prime}+L^{\prime}\right)^{2}} ; \quad T_{0}=\frac{1}{6}\left(\frac{L^{\prime}+3}{L^{\prime}+1}\right)=\frac{D t_{0}}{l^{2}}
$$

and

$$
\frac{N_{1}(b, t !}{C_{0} A l}=\left(\frac{L^{\prime}}{I+L^{\prime}}\right) T\left(\frac{L^{\prime}}{I+L^{\prime}}\right) T_{0} \varphi_{4}\left(L^{\prime}, T\right)
$$


Substinstion (1) Eq. 125$)$. Eq. (26). and the definition $T=D / R^{2}$ into Eq. (23d):

$$
\left.F R(1)=m i \quad m t_{0}\left|r_{s}\right| L^{\prime} \cdot T\right) \mid
$$

whith stales an interesting result. So long as $r_{0}$ is independent of time, all experimental retease curves wblaned with a lime constant $C_{0}$ have the same general shape with or without film effects. They coasisi of a straight line originating at a maximum lag time, $t_{0}$. with dispersion effects characterized by premature breaklhrough in degrees determined by $\varphi_{4}\left(L^{\prime}, T\right)$.

\subsubsection{Difiusion Comtrol - No Fum}

At this limit. $h^{\prime}$ (and ihus $L^{\prime}$ ) approaches infinity. Then. using Eqs. (25) and (26). One can show that

$$
T_{0} \rightarrow \frac{1}{6}
$$

and

$$
C_{0} \rightarrow \frac{M_{i m l}^{0}}{A D}=\frac{M_{i}^{0} \mathrm{mbl}_{0}}{A i}
$$

Equations (23j) and (27a) become:

$$
\frac{N_{1}(b .1)}{C_{0} A l}=T \quad \frac{1}{6} f_{4}(\infty . T)
$$

and

$$
\left.F R(t)=m i \quad m t_{0} \mid \varphi_{3}(\infty . T)\right]
$$

The Iransient parameter $f_{4}$ approaches the well-known series form obtained from the specialized D controlling derivation outlined by Ciank. ${ }^{16}$ One finds that

$$
{ }_{4}(\infty . T)=1+\frac{12}{\pi^{2}} \sum_{1}^{\infty} \frac{(-1)^{n}}{n^{2}} \exp \left(-n^{2} \pi^{2} T\right)
$$

as specified in Fig. 7.

We have found that the normalized relationship. Eq. $(23 \mathrm{e})$, is very convenient for presenting results $n i^{\circ}$ several related experiments on a single plot. Figure 8 illustrates the shape of this curve, along with that of a "typical" experimental curve that would result if $t_{0}=500 \mathrm{hr}$ and $\mathrm{m}=1.5 \times 10^{-5} / \mathrm{hr}$. From the intercept. one obtains the diffusion coefficient using

$$
D=\frac{1}{6} \frac{l^{2}}{t_{0}}
$$



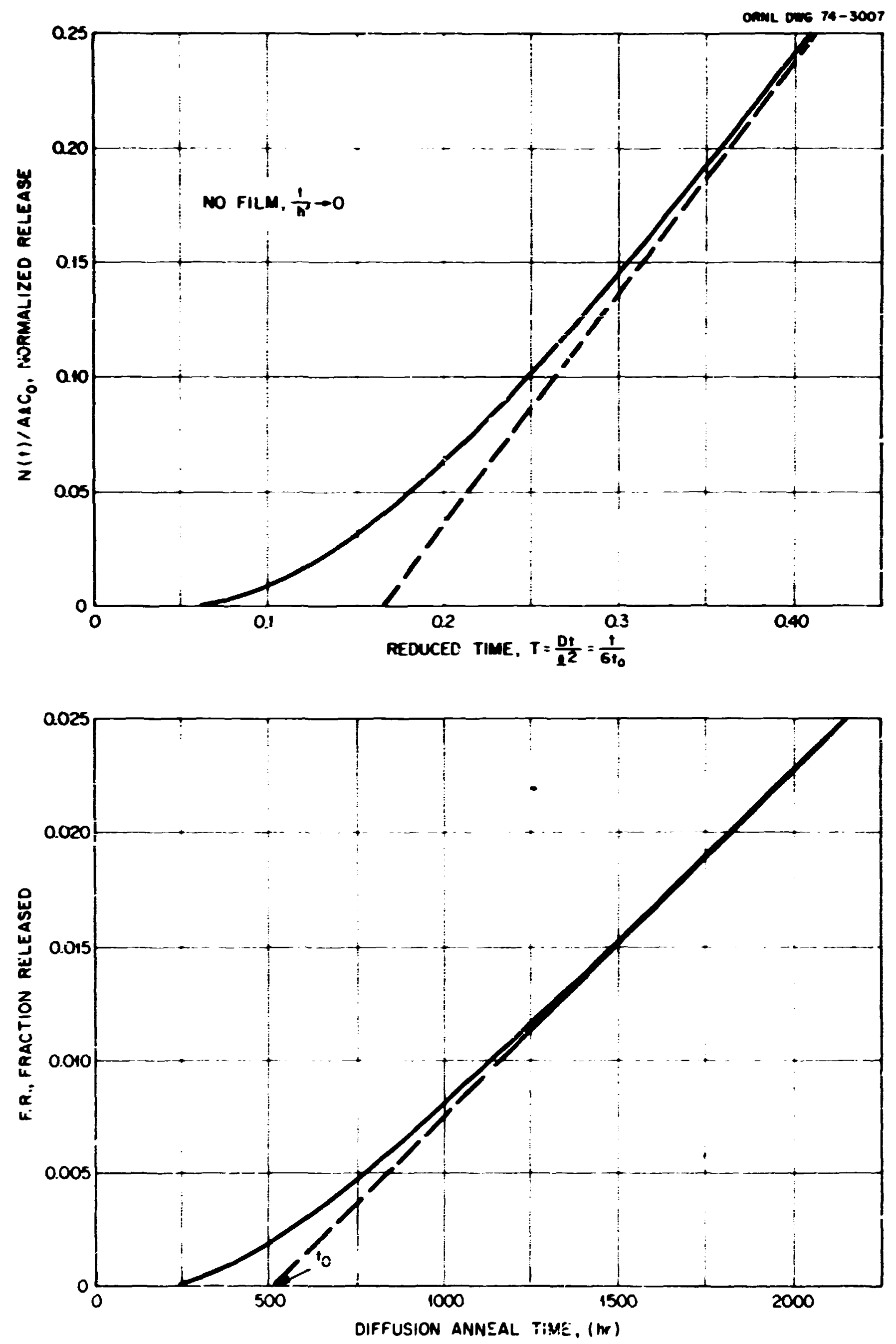

Fig 8. Normalized and Experimental Release Curves During Approanh to Steady State. These curves snow transient effects as they appear on restangular coordinates for a constant-potential system with no illm effects. 
From the slop: orie ubtiolus the driving potential using

$$
C_{0}=\frac{M_{1}^{\prime \prime} m r_{10}}{A l}
$$

where $A=4 \pi$ ah in spherial courdinates. A knowledge of $i$ is not required (') use these relationships. If it were known and applied in the $C_{c}$ expression in tine proper iashion. it would simply cancel out .

\subsubsection{Diffusion with Fam Effects}

It is of interest (1) consider (1) how film effects might influence the shape of an experimental release curve. ind $(2)$ the degrees of unce:tainties in $C_{0}$ and $D$ introduced $b_{0}$ ignoring their possible presence. We shall assune that film measurements are available. but not to the investigator correlating release data. For disiussion. we consider three cases: (!) a total concentration decrease sicurs across the madium (rio film); (2) $y_{3} C_{0}$ arross the mediun: $1 / 3 C_{0}$ across a film: and (3) $1 / 3 C_{0}$ across ate medium. $2 / 3 C_{0}$ across a film. Respeciive $L^{\prime}$ values would be $\infty .2$. and $1 / 2$. Plots of the normalized release prepared on the basis of $E q$. (23d) and the interpolated $\varphi_{4}$ values fron Fig. 7 are shown at the 10, of Fig. 9. Each $L^{\prime}$ case has a separate and distinct normalized plot. ultimate slope. and $T_{0}$ intercept value.

Nexi. a predicted experimen!d curve for each case was computed uing the same infcimation and Eq. (273). One may see the results at the bottom of Fig. 9. Computed release-vs-time values for all cases feli precisely on the same curve. (Only at very iow L' values would some effects be seen.) The unwary experimenter would have no knowledge that a film was influencing his results; he would simply proceed under the assumption that $\mathrm{L}^{\prime} \rightarrow \infty$ and would compute $\left(C_{0}\right)_{\text {app }}$ and $D^{*}$ yalues accordingly. Errors in $D$ would he gross. as indicated by Eq. (26). For $L^{\prime}=2$ and $1 / 2$, the true $D$ should have been $5 D^{*} / 3$ and $7 D^{*} / 3$. However. even with the wrong value for $D$. estimates of $C_{0}$ would be quite acceptable since with an approximate $D^{*}=\left(\ell^{2} ; 6 t_{0}\right)$.

$$
C_{0}=\frac{M_{1}^{0} m\left(\left(L^{\prime}+1\right)^{2}\right.}{A D^{*} L^{\prime}\left(L^{\prime}+3\right)}=\frac{\left.\left(C_{0}\right) \mu p p^{\left(L^{\prime}\right.}+1\right)^{2}}{L^{\prime}\left(L^{\prime}+3\right)} .
$$

Thus. at $L^{\prime}=2$ and $1 / 2 . C_{0}$ would be $9 C_{0}^{*} 10$ and $9 C_{0}^{*} 7$. In iact. if $L^{\prime}=1 . C_{0}=C_{0}^{*}$. We conclude that film effects must be determined by direct measurement of concentration profiles. however. even in the absence of such iniormation. good estimates of $C_{0}$ can be obtained assuniing $\mathbf{L}_{-} \rightarrow \infty$.

\subsubsection{Approximation of Deplecion Curves}

In many cases. the diffusion-controlled constant-potential correlation is applied to experimenial curves that evidence jepletion effects at terminal regions of the time scale investigated. Some question arises as to the interpretations of $D$ and $C_{0}$ thus obtained. That is. if depletion were indeed occurring throughout the experiment. would these parameters have physical significance? Now, a theore:icai slope $m$ ' and diffusion coefficient $D^{\prime}$ may be acquired from Fig. 5 at a given $L$. A reiationship i "ween the theoretical and approximate slopes. $\mathrm{m}^{\prime}$ and $\mathrm{m}$ respectively. is:

$$
\mathbf{m}=\left(f T_{0}^{\prime} / t_{0}\right) m^{\prime}
$$



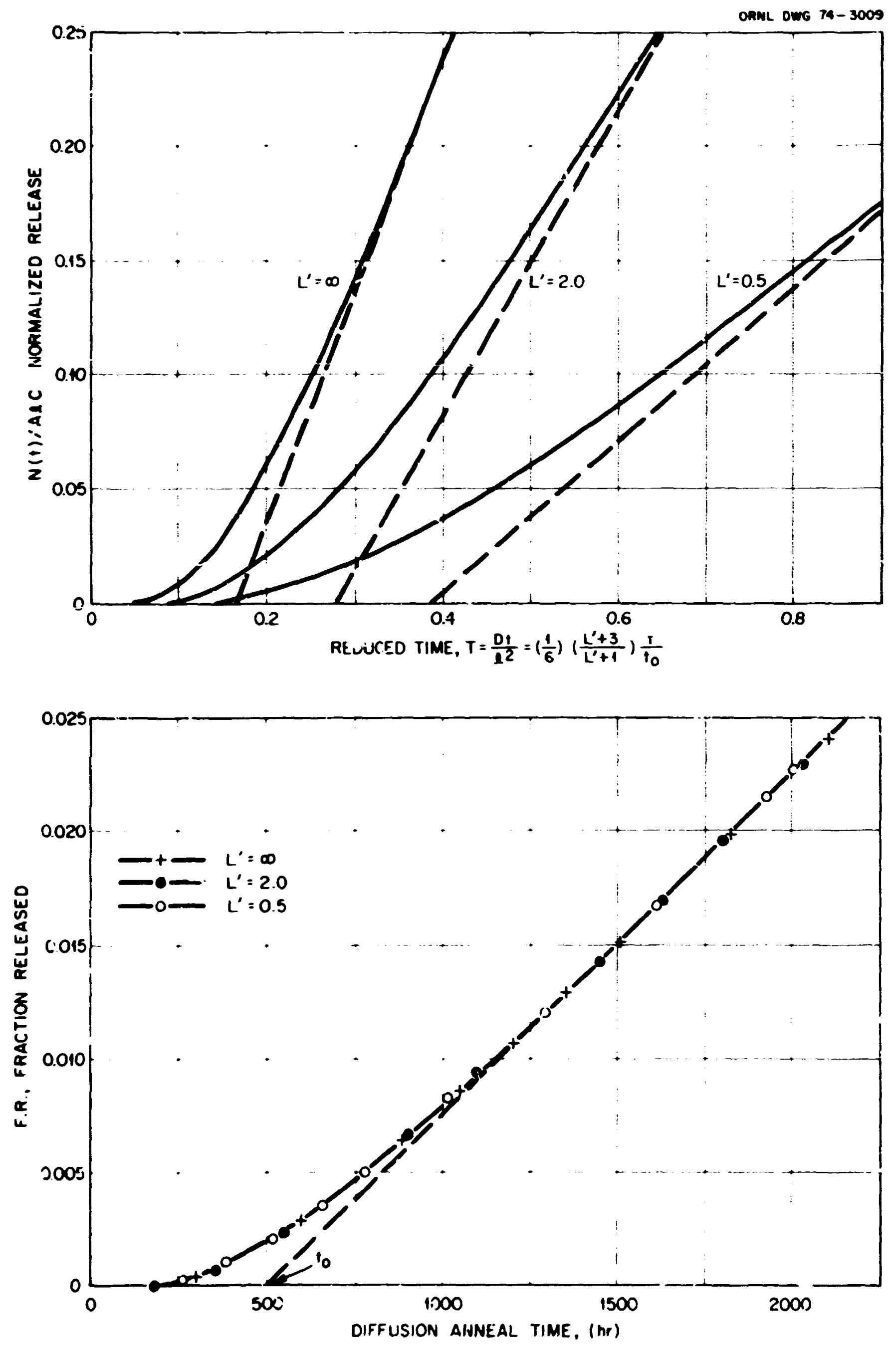

Fig. 9. Effect of Films on Ptormalized and Experimental Release Curves. ilthough norma.'ized curves .ire drastically different, the experimental curves would be nearly identical. 
The constant-potential correlation gives:

$$
C_{0}=\frac{I M_{1}^{0} I^{\prime}}{A l} \frac{i_{0}^{\prime}}{T_{0}}
$$

where

$$
T_{0}=1 / 6 \text {. and } T_{0}^{\prime} \text { is taken from Fig. } 5 .
$$

The true runconstant potential is obtained from Fig. 6 :

$$
C_{2}(a, t)=\left[\frac{C_{1}^{*}(i)}{C_{1}^{*}(0)}\right] \frac{\mathrm{MH}_{1}^{0}}{V_{1} \phi}=\left[\frac{C_{1}^{*}(t)}{C_{1}^{*}(0)}\right] \frac{\mathrm{r} M_{i}^{0} L}{A l}
$$

since

$$
\dot{q}=A l / V_{1} L
$$

The ratio between the approximate $C_{0}$ and $C_{2}(a, t)$ is:

$$
\frac{C_{0}}{C_{3}(a, t)}=\frac{T_{0}^{\prime} m^{\prime}}{T_{0} L}\left[\frac{C_{1}^{*}(0)}{C_{i}^{*}(t)}\right]
$$

All p"-ameters here are "theoretical." With this formula, it can be shown that the $\mathrm{C}_{0}$ estimate is, in fact. a gcod approximation of the tue $\mathrm{C}_{2}(\mathrm{a}, \mathrm{t})$ prevai:ing at the tirie $\mathrm{m}$ was evaluated. The true coefficients range from $(0.6) D^{*}$ to $D^{*}$, where the latter is derived from tite $1 / 6$ approximation.

\subsection{Depletion After Constant-Potential Operation}

\subsubsection{Linear Flow Solution}

The approach and :echniques used here are quite similar to thos: used for the depletion relationships derived in sect. 3.1 - except that the initial conditions are modifies. Here we take $t^{\prime}$ as the new time scale. The new zero time is selected at the point at which the release data show a distinct departure from "straight-line behavio.."

The initial conditions are:

$$
C_{1}(C)=C_{1}^{*}(\tau) . \quad C_{2}(a, 0)=C_{2}(\tau),
$$

and

$$
C_{2}(x, 0)=C_{2}(\tau)[(b-x) / l]
$$

The symbol $\tau$ is a point in old time corresponding to $t_{0}^{\prime}=0$. If the total release up to $\tau$ has been measured, the active amount in the source zone can be computed with a knowledge of $f$ and the steady-state coaing content. 
The boundary conditions. in transformed coordinates. are:

$$
\begin{aligned}
& c_{2}(b, s)=0 . \\
& c *(s)=s \dot{c}_{2}(a, s) .
\end{aligned}
$$

and

$$
s c_{1}^{*}(a, s)-C_{1}(+0)=\left(D A / V_{1}\right) \frac{d c_{2}(a, s)}{d x}
$$

Application of all ith abore to

$$
\operatorname{sc}_{2}(x, s) \quad C_{2}(x+0)=D \frac{d^{2} c_{2}(x, s)}{d x^{2}}
$$

yields:

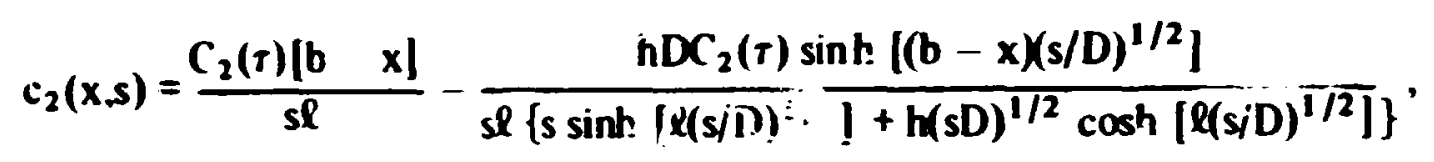

where

$$
h=A / V_{1} \phi=1 / \mathbf{a}^{\prime} \phi
$$

The inverse of the first term is canceled out by the inverse of the $s=0$ pole of the second term. The answer comes from setting

$$
i a / l=(s / D)^{1 / 2}
$$

and evaluating the complete second term, as dictated by Eq. (1!). The result is:

$$
C_{2}(x, t)=C_{2}(r) \sum_{1}^{\infty} \frac{2 L^{2} e^{-\alpha_{n}^{2} T} \sin \left[\alpha_{n}(b-x) / l\right]}{\alpha_{n}^{2}\left[L(L+1)+\alpha_{n}^{2}\right] \sin \alpha_{n}}
$$

where $\alpha_{n}, n=1,2,3 \ldots$ are the positive roots $\mathrm{c} i$

$$
\alpha_{n} \sin \alpha_{n}=\text { : }
$$

and

$$
L=l h=\frac{l A}{V_{1} 6}
$$

when $x=b, C_{2}(x, t)=0$, as it should. When $x=a$, 


$$
\frac{c_{2}(a, t)}{r_{2}(\tau)}=\frac{c_{i}^{*}(t)}{c_{1}^{*}(\tau)}=\sum_{1}^{\infty} \frac{2 !^{2} c^{a_{n}^{2} T}}{\left.a_{n}^{2} i u(L+1)+a_{n}^{2}\right\}}=1 \quad r_{3}(L . T)
$$

Formation of the flux at $b$, followed by integration. gives:

$$
N(b . t)=\sum_{1}^{\infty} A \frac{l^{2}}{D a_{n}^{2}} \frac{2 h D C_{2}(\tau)\left(l-e^{-a_{n}^{2} T}\right)}{\left.\mid(h l)^{2}+(h l)+a_{n}^{2}\right] \cos \alpha_{n}}
$$

Development of the proper fractional release expression for times greater than $\tau$ requires a special base value that includes both source anc coating contents. We iecall that $h=!, a^{\prime} \phi$. Then.

$$
\begin{aligned}
\text { Total active content } & =[\text { content (kernel }+ \text { buffer })]+[\text { content (coating) }], \\
& =\mathrm{C} \text { [area of a box }+ \text { area of a triangle] }, \\
& =\mathrm{aAC}_{1}^{*}(\tau)+\frac{\mathrm{A}}{2} \mathrm{RC}_{2}(\tau) \\
& =\mathrm{AC}_{1}^{*}(\tau)\left[\mathrm{a}^{\prime}+\frac{\ell}{2 \phi}\right]=\frac{\mathrm{AC}_{\mathrm{l}}^{*}(\tau)}{\phi}\left[\frac{2+\ell \mathrm{h}}{2 \mathrm{~h}}\right] \\
& =\frac{\mathrm{AC}_{2}(\tau)}{\mathrm{h}}\left[\frac{2+\ell \mathrm{h}}{2}\right]
\end{aligned}
$$

The ratio of $M(b, t)$ to the above is:

$$
\left.F\right|_{\text {after }}=1-\sum_{1}^{\infty} \frac{4 L^{2}\left(1-e^{-a_{n}^{2} T}\right) \sec a_{n}}{(L+2) a_{n}^{2}\left[L(L+1)+a_{n}^{2}\right]},
$$

or

$$
\left.F R\right|_{T}=\hat{f}_{4}(L . T)
$$

Again, we encounter functions covered by Carslaw, 15 Jaeger. ${ }^{14,15}$ and Clarke. ${ }^{14}$ It should be understood that the fraction-remaining function here in terms of measured $F R(t)$ is:

$$
\left.F R\right|_{\tau}=\frac{F R\left(t^{\prime}\right)-F R\left(t_{0}^{\prime}\right)}{F R(\infty)-F R\left(t_{0}^{\prime}\right)}
$$

\subsubsection{Discussion}

Generalized curves for this function are shown in Fig. 10, where $T$ is plotted on an exponential basis. Figure 11 shows the results of transposing these curves to rectangular coordinates. In many ways, the curves reflect the same cha:acteristics of those in Figs. 4 and 5. However, there is one important exception. All curves in Fig. 11 proieed directly toward the origin. There is no "delay time" because the coating has been "preconditioned" with a sieady profile at time $t_{0}^{\prime}=\tau$. 


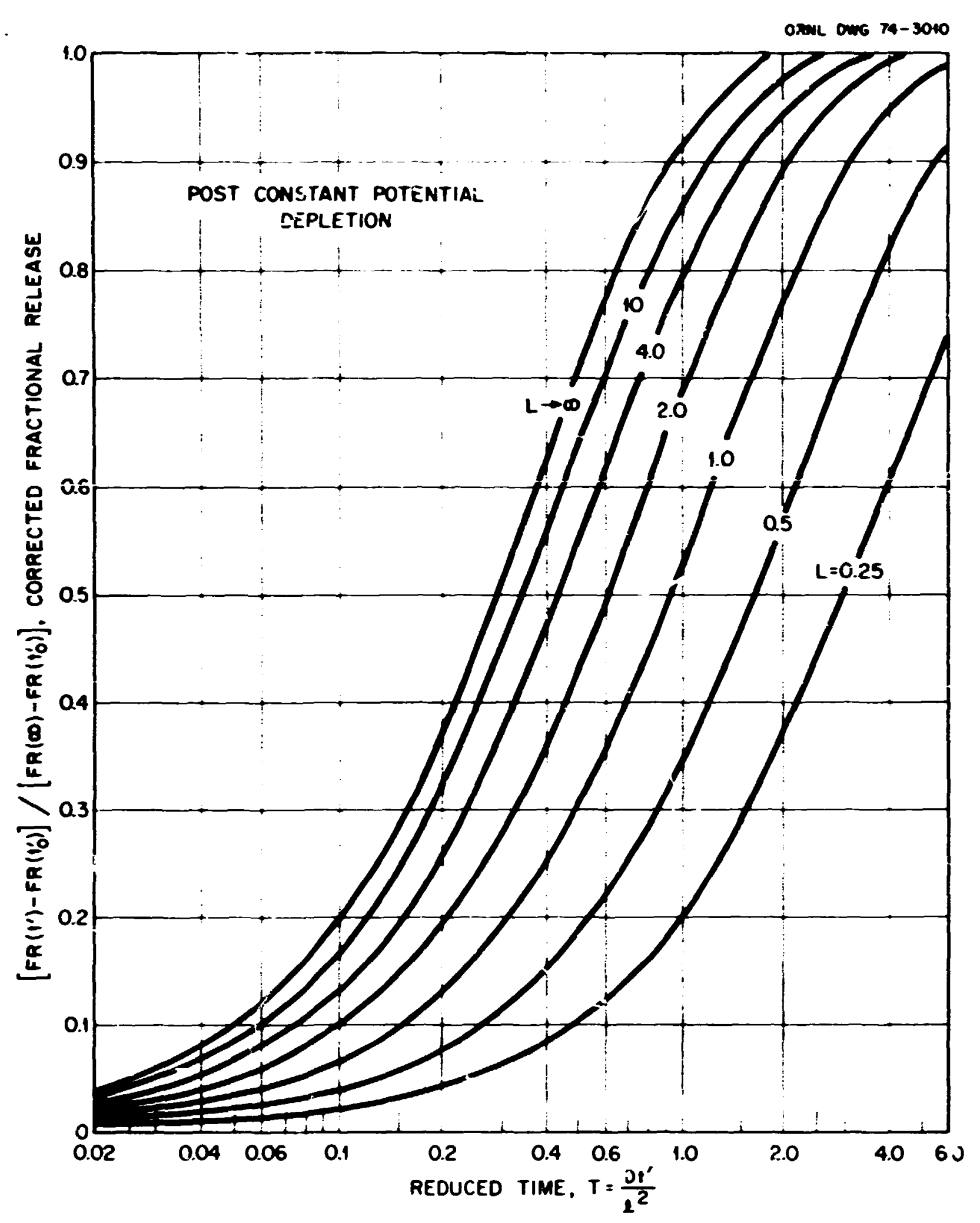

Fis 10. Depletion Curves Aseocinted with Equation (35b). The release function represents behavio: anticipated for Region C. Figure 1. when the coating concentration starts to decrease after a period of constant-potential operation.

In an effort to evaluate these results. we have fitted Eq. (35b) to the most controversial data at hand. namely, those of Curve I. Fig. I. The results are presenitd in Fig. 12. As seen, there is a "right" intercept at about $17 \mathrm{hr}$ which agrees with the bulk of our cata int that a coefficient derived in this manner is acceptably high. On the other hand, some event ocisirring at about $75 \mathrm{hr}$ caused the curve to rise in an inexplicable tashion at a very high rate. Present speculations attribute this strange behavior to an internal failure of the kernel; for example, an appreciable portion of the trapped fraction may have broken free at $75 \mathrm{hr}$, impinging a new, higher drivirig iorce on the system. The coating did not fail because noble gas retention was maintained. 


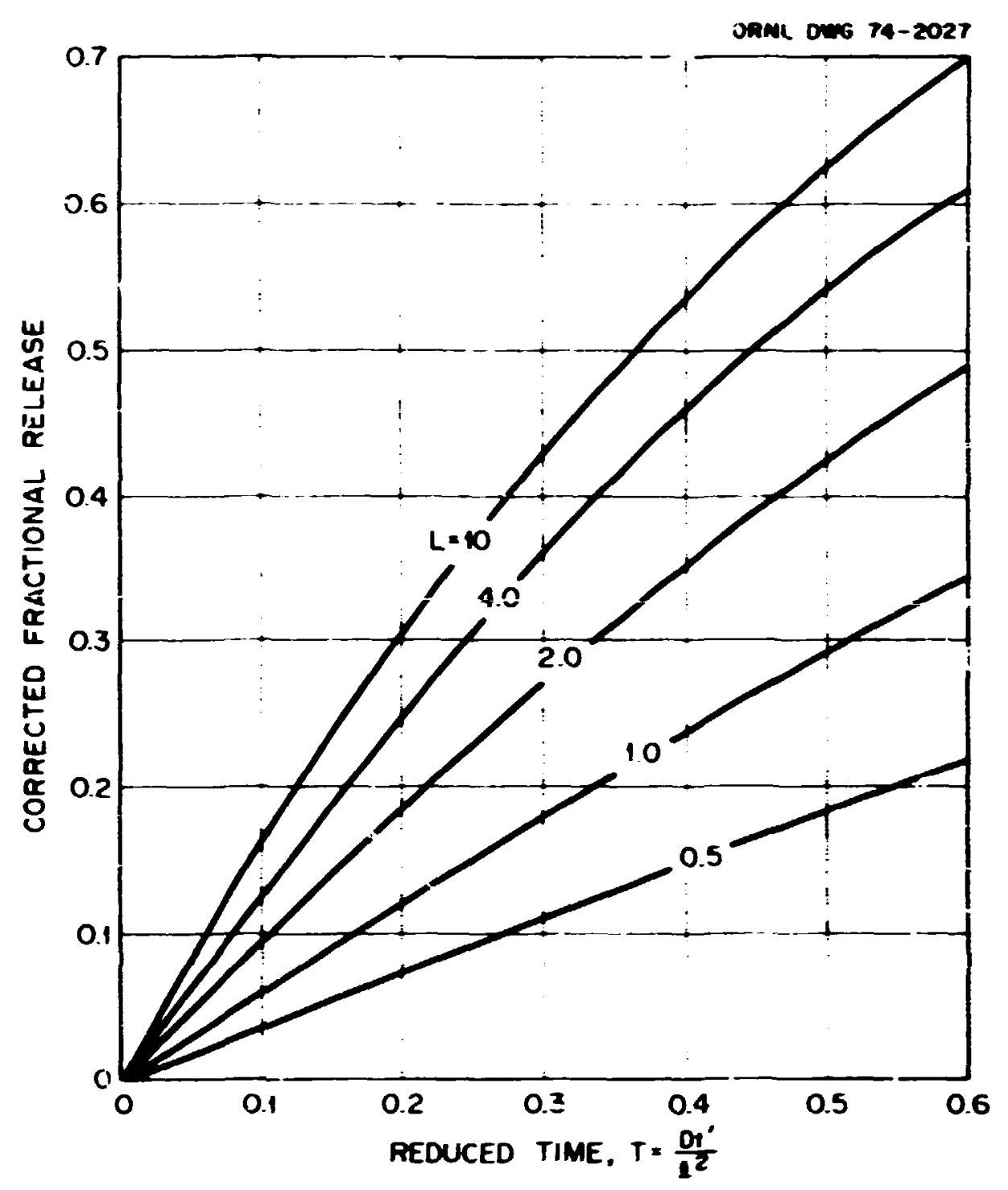

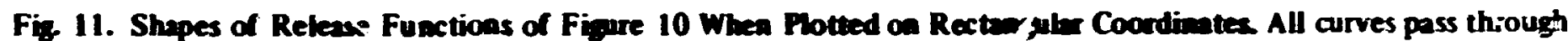
the origin.

Clearly, the system was not at constant potential, but it was subject to an initial profile when depletion took over. We arbitrarily set this time at $195 \mathrm{hr}$, then fitted Eq. (35b) tc the remainder of the curve. To ubtain the fit, it was necessary to assume that $D$ corresponded to $45 \mathrm{hr}$ and $\mathrm{L} \rightarrow \infty$. The decay in this case seems to follow a special "thin:iaysi" depletion rharacterized by a zero-source concentration. Rough back-calculations suggest that the source zone became depleted of cesium somewhere between 100 and 200 hr. In any event, Eq. (35b) does give a description of $\cdot n$ ultimate decay mode.

\section{AUXILIARY DETERMINATIONS}

\subsection{Experiment: with Separated Particle Components}

We have mentioned the importance of performing anneais with bare kernels in order to obtain rapid measurements of $f$ for correlation of release data from intact coated particles in depletion modes. Use of this approach presumes that all trapping effects occur in the oxide; it also inplies that kernels can be removed intact from cracked particles. There may be considerable merit in proposing attempts to separate all three components - kernel, buffer, and ccating - for rough determination of overall fission preduct distributions. One might also perform individual anneals to assay relative retention characteristics At least, these anneals would pinpoint the region where permanent trapping occurs. 


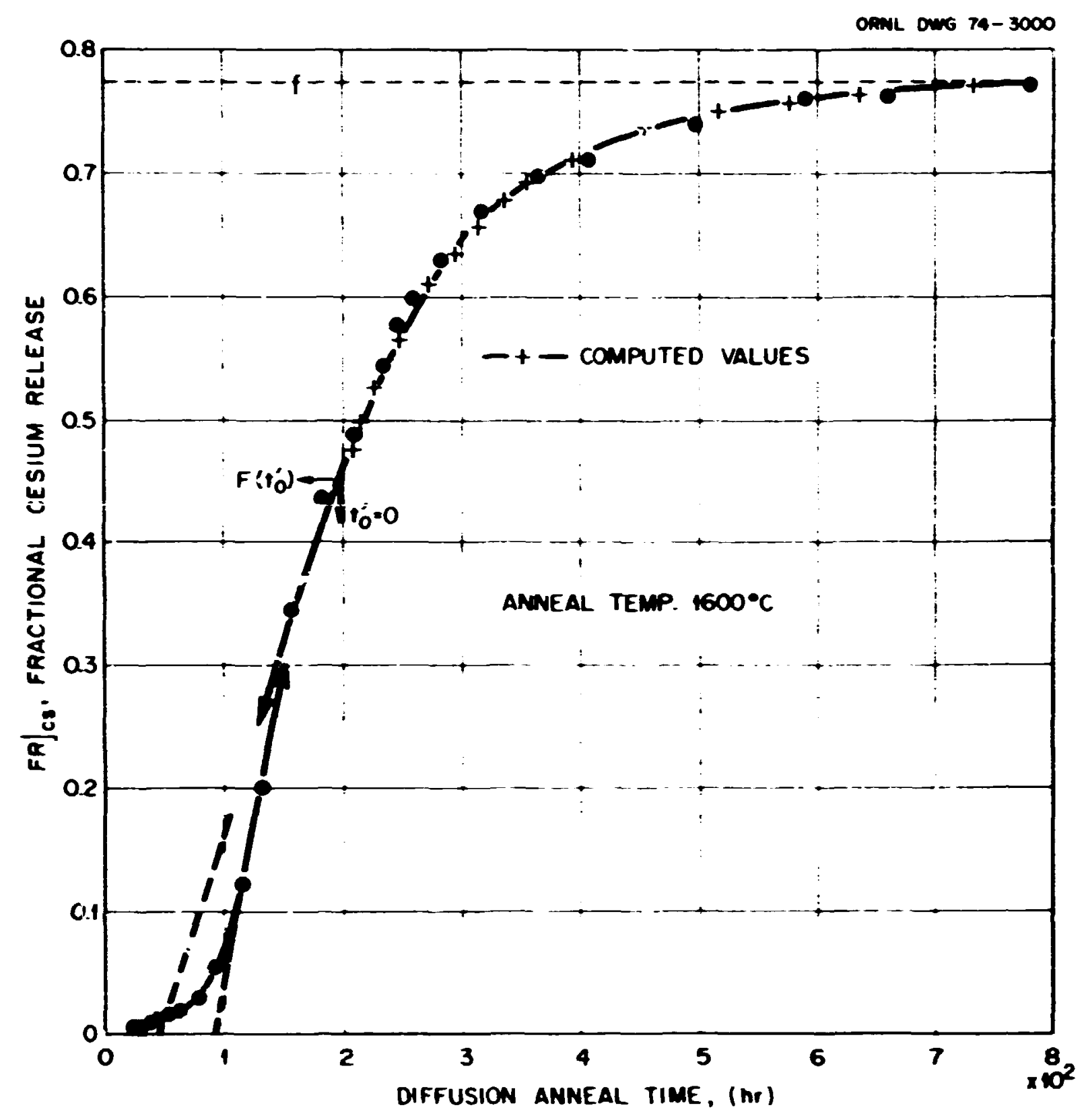

Fiz 12 Application of Equation (35b) to Rejon C, Curve 1, Fipre 1.

Infurmation sought here would not be of the nature of "fine structure" but would consist of good average values to clarify major experimental features such as the controiling modes of release at the time the particles were cracked. For example, if coatings of particles sampled at different times during an anneal experiment had the same fission product contents, a constant-potential mode would be verified.

Unfortunately, component separation is a very tedious procedure, its success depending on the relative hardness of the components and the degee to which they are bonded together at room temperature. Kernels are the most easily extracted. If buffer material adheres to the kernel, it can be removed with mild abrasive action. Separation of buffer and coating is the most difficult operation of al!. In addition, there is the problem of recovering all debris to obtain suitable aliquots. In most cases, the only recourse is to attempt layer-by-layer removal of the coating until the buffer-coating imteiface is approached. For this and other reasons, we unust concede the necessity of grinding coatings.

\subsection{Concentration Profiles by Grinding}

Nearly all the classical "solid-state" diffusion expe:imenis are arranged such that one or two species (in compatible substrates) will mix in a predictable fastion as reflected by the shape of concentration profiles in the substrates. All volatile species represent special cases requiring special techniques. However, most solid-state studies are roncerned with slow-moving nonvolatile species. If, for example, one desires to make 
self-di!fusion measurements of radioactive silver in polycrystalline silver, an experiment can be devised as lollows: A very thin layer of tracer is placed on the substrate (as a Dirac delta function); the substrate is then annealed at various temperatures to form decay patterns [given by $C(x, i), i$ fixed $j$; and, inally, the patterns are fitted to the function

$$
\frac{C(x, \bar{i})}{C(0, \bar{i})}=\exp \left(\frac{x^{2}}{4 D i \bar{t}}\right)
$$

The log of activity (proportional to the concentration ratio) is plotted vs $x^{2}$ to evaluate $D$, which is proportional to the slope of the curve. The ultimate objective is to obtain D's at several temperatures, plot these as a function of $\left(T^{\circ} K\right)^{-1}$, and inake some observations concerning the slope of this curve - since it represents an activation energy. While this is an acceptable goal, our overall problem is quite different and much more complicated. We cannot arrange cesium experiments to fit the silver pattern if we have high cesium concentrations in the source and measure vapor evolution at $r=b$. But solid-state theoreticians feel ciomforted if such data are available. Let us evaluate the utility of grinding data as it will apply to our experiments. It should be noted here that results of an exhaustive study based on profile shapes have been reported by Baurmann. ${ }^{17}$

We consider any particles that are removed from an anneal to be in a depleting mode. Unless they are obtained during the very initial stages of the anneal, corresponding profiles will show only rather indifferent variations in curvature as influenced by "smeared-out" contributions of several variables. They will not display the clear-iut (maximum) transient behavior characterisix of the silver experiments. To assure ourselves that this would be the case, concentration functions for the depletion cases were manipulated to form integrated grinding equations which give the cumulative amount removed after grinding fiom $b$ to $r$. Ihe analytical results are too complex to present here; however, a glance at the results reveals that the shape of release curves - not the shapes of grinding curves - would provide the best means for estimating D and $\phi$. Aside from rugh rerification by profile shaps, $\phi$ might best be determined by performing a straightforward extrapolation of profiles to $r=a$ in orcier to estimate $C(a)$, and then combinirg this information with concentration measurements in the source to determine directly.

Of course. profile information might be employed in an auxiliary role, particularly when the effects are hidden in the release data. One example is determination or verification of initial conditions; another is detection of elusive boundary conditions as epitomized by film effects. We consider the latter. The most suitable specimens for a study of this type would be found in a release experiment operating in the constant-potential mude with demonstrated steady-state release. Under these conditions, a fair estimate of $C_{0}$ can be made even if $D$ (from lag time) is in error. Regardless of $D$, the general steady-state implies that:

$$
J A=N^{\prime}(t, a l l r)=(A) C_{a}\left(\frac{h^{\prime}}{L^{\prime}+1}\right)
$$

The prime on $\mathbf{N}$ denotes $\mathbf{d N} / \mathbf{d t}$, which is constant in this case.

There are two decreases in concentration: one between $r-a$ and $b$, and a second (to zero; beiween $r=b$ and $b+\delta$, where $\delta$ is quite small. If we can evaluate the extent of the first decrease, we car calculate the second and determine $h^{\prime}$. Over the region of the first decrease, one may express the rate in rifferential form as:

$$
N^{\prime}(t)=-4 \pi r^{2} D \frac{d C}{d r}
$$


Integration gives:

$$
C(r)=C_{j}-\left(\frac{1}{a}-\frac{1}{r}\right) \frac{N^{\prime}(t)}{4 \pi D}
$$

or

$$
N^{\prime}(t)=\frac{D(4 \pi a b)\left(C_{b}-C_{a}\right)}{(b-a)}=\frac{D(A) \Delta C}{l}
$$

Thus,

$$
C(r)=\left[C_{2}-\frac{\dot{b} \dot{A} c}{l}\right]+\left[\frac{a b \Delta C}{l}\right] \frac{i}{r}
$$

This is the profile expression that we have alluded to all through this discussion, as if we had at our disposal some undisclosed means for measuring point concentrations directly. Of course, such means are actually available (e.g. microprobes). Yet, these fiequer:tly give too much detail (see jagged line in Fig. 2 ;. and the problem of tying in absolute values to scans is left to the investigator as an expensive calibration exercise. Another profile expression in spherical coordinates may be deiived from Eq. (13).

If grinding information is available, one can "estimate" profiles by dividing the count data of an ircrement by the average increment volume; unfortunately, however, the "tying in" problem remains. It becomes necessary to accept the fact that integrated quantities must eventually be used, although estimated profiles are obviousiy useful in a generd sense.

We write an equation for the amount removed after grinding, volumes $\Delta V$ away from $r=b$ back to any $r$ between b and a, as follows:

$$
\int_{V_{r}}^{V_{b}} C(r) d V=\left[C_{2}-\frac{b \Delta C}{l}\right]\left(V_{b}-V_{r}\right)+\frac{(A) \Delta C}{2 l}\left(b^{2}-r^{2}\right)
$$

where $(A)=4 \pi a b$. Now if we let $V_{r}=V_{a}$, the integral refresents the correct (VC) area under the entire profile. This corresponds to the cumulative count of the coating. Firally, we form the fract $t^{\circ}$, $n$ of total removed, let $\Delta C=\gamma C_{a}$, and draw up a set of these curves as a function of $\gamma$. Comparisons of the experimental fractional curves with computed values should give $\gamma$ if $C_{2}$ is known. Thus $C_{b}$ and. in turn, the value of $h^{\prime}$, can be determined.

Some predicted experimental curves involving profile information, as discussed above. are shown in Fig. 13. Both linear flow in slab geometry and radial flow in spherical coordinates have teen covered. There are significant differences in the two sets of curves due to the difference in the geometry of the flow pattern. The concentration profiles are shown at the top of Fig. 13. Those for slab genmetry are straight, while thcse for spherical geometry are concave (except where $\gamma=0$ ) and fall below the linear curves. The curves for the fraction remuved are shown at the bottom of Fig. 13. The curves for the linear system are concave (except where $\gamma=0$ ), while curves in the spherical system are both concave and convex. Neither set of fraction-remaining curves provide much detail since the lines fall close together in the range $\gamma=0$ to 1 .

Since experimental grind:ng data may be somewhat scattered, it would seem most practial to smooth the data by plotting the fraction removed vs coating thickness and then to dif.srentiate and obtain the concentration ratios for comparison with theoretical curves to determine $\gamma$. 

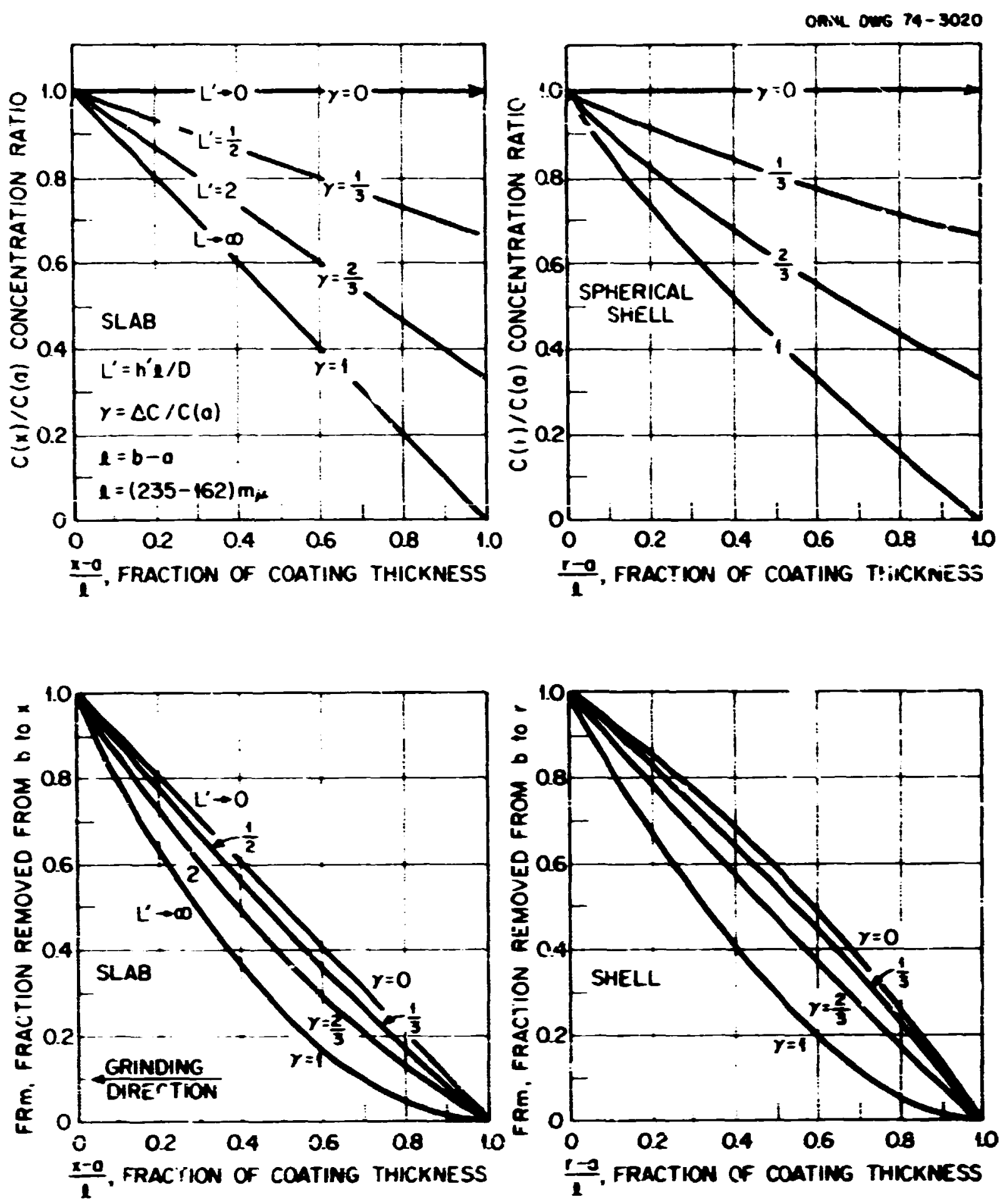

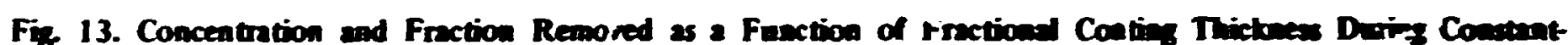
Potential Releave. Curves for both linear and ratial (spherial) systems are stown to illustrate effects of filus [see Fig-9].

\section{Sumnavy}

Separation of components of coated particles with subsequelı: analyses will provide cesium inventories which will aid in the interpretation of release data and grindirg results. The separated components may then be annealed as a rapir method of determining $f$, the available fraction of cesium. Kernels recovered from grinding operations may also be annealed to determine $f$.

The auxiliary grinding experiments are considered useful to obtain gross internal detail to complement the release experiments. particularly to explore the effects of films. Grinding is the only method by which information on films may be obtained. In the event that grinding techniques can be refined to provide sufficient detail to give meaningful results, they should be presented in cerms of concentration profiles obtained by $g$ raphically differentiating the integral cu:res. Correlations must be carried out with equations developed in terms of spherical geometry. Only the release data may be compared with solutions obtained frori slab geometry. 


\section{CLOSURE}

Diffusion equations applicable to the release of cestum from pyrocaibon-coated fuel particles were reviewed and/or derived reiative to possible initial and boundary conditisns which might exist in postirradiati.un annealing experiments. Limited evidence is presented to show that buffer and kernel regions may be grouped together as one source region for the purpose of simplifying the sa!culations. Equations for diffusion governed by constant potential, by depleting potentiai, and by a hybrid situation involving both conditions were considered.

Since diffusion equations for a depleting potential in spherical geometry are exceedingly complex, solutions were compared with similar, simpler equations for slab geometry with dimensional parameters adjusted to correlate with spherical dimensions. Results were shown to be sufficiently equivalent so that linear equations may be used in calculations of release, but significant differences were noted in calculations of concentration profiles for linear and spherical systems, applicable to grinding experiments.

Experimental evidence was presented it show titat some cesium is permanently trapped in the kernel. The trapped fraction has no effect on the releast when a constant potential is maintined but must be considered when the driving potential is changing (e.g., depletung potentzal).

The bre:-ithrough time (for cesium to penetrate the coating) determined from the intercept of the linear part of a release-vs-time plot of a constant-potential experiment is used to calculate the diffusion coefficient, $D$. It was shown that breakthrough times determined from intercepts of near-linear portions of curves in depleting potential experiments, if used in the constant-potential relation, $D=l^{2} / 6 t$, would provide close approximate values of $D$.

It was found that film effects are not apparent in release experiments; the film coefficient and the diffusion coefficient may be combined- in an apparent $L^{*}$. Separate values car be obtained only from concentration profile experiments. Release data may be used to calculate the apparent diffusioni ioefficient, $D^{*}$; the trapped fraction, $(1-f)$; the driving potential. $C_{2}(a) ;$ and the partition coefficient, $\phi$. Accurate values of $D^{*}$ and $C_{2}(a)$ are obiained only from constant-potential experiments. Depleting-potential curves are required tn obtain $\phi$. and will also furnish approximate $L^{*}$ vaues and an average $C_{2}(a, t)$. The available fraction, $f$, must also be known in order to calculate $\phi$. When the time required to exhaust the source is excessive. $f$ and $\phi$ may be obtained from depleting potential experiments by manipulating the values of $\hat{i}$ and $\phi$ to obtain a fit with calculated curves; however, the exrerimental curve of release vs time must include at least the beginning of the second break of the S-shaped curve. A better approach would be to obtain $f$ by annealing bare kernels and then use this informacion, along with the release curve, to compute $\phi$.

Finally. wo note that the depleting potential implies a limited source and. therefore, would be most applicable to coated particles with low burnup. The constant potential dictates that the source be unlimited with respert to the concentration in the outer coating. This requirement may be met if the source concentration is kept above a certain value for the duration of the constant potential regime, and would be most likely to occur in high-burnup coated particles.

A constant potential may be sustained under twc conditions:

(1) A constani vepor pressure above excess fission products or fission product compounds in the source zone.

(2) A limiting concentration or "apparent" solubility in the coatıng such as might be attained when ail temporary trapping sites (defects) are occupied in the coating rexi to the buffer-coating interface.

The first condition will produce a change in potential with temperature, while the second is temperature independent. Annealing, and frinding experiments to obtain informatior on actinide diffusion have produced the temperature-independent limiting concentrations indicated in (2). Average solubilities in 
columiar py rocarbon determined from 13 experiments with uranium and fron $\$$ experiments with thorium were found to be essentially constant at 1.26 and $1.38 \mathrm{mg} / \mathrm{cm}^{3}$, respectively, over the temperature range 1553 to $2065^{\circ} \mathrm{C}$. Barium and strontium are known ${ }^{18}$ to segregate as oxides in $\mathrm{UO}_{2}$. If constant-potential release of barium and sincistium occurs, it would probably be of the type indicated in (1). Cesium compounds are less sianle and highly volatile at high temperatures; the refore. limiting solubilities are more likely to control in this case.

\section{ACKNOWLEDGï̀NENTS}

The alithors are greatly inc'ebted to C. W'. Nestoi, Computer Sciences Division, for explaining and demonstrating the applications of the inversion integral. Appreciation is also expressed to H. J. de Nordwall for his iselpfu! suggestions, advice, and guidance through this project, and for his critical review of the maruscript. Speciai thanks aie extended to A. P. Malinauskas, wi: anzint ained a high lky:ol of interes: and was a constant sou,ce of encouragement duririg the research. Finally, we acinowledge the invaluable assistunce of Martha Stewart, who edited the manuscript, and of R. D. Cheverton, who served as a technical reviewer.

\section{NOMEP.CLATURE}

$a^{\prime} \quad$ Volume-t(1)-area ratio. $\mathbf{V} / \mathbf{A}$, at the source zonc-coating radius, $\mathrm{cm}$

2 Position of source zone-coating interface, $\mathrm{cm}$

A Area normal to diffusive fluw, $\mathrm{cm}^{2}$

$A(s) \quad$ Constant of integration in "s" space. atom-sec $/ \mathrm{cm}^{2}$ or atom-sec/cm

b Outer boundary of coated particle. $\mathrm{cm}$

As a subscript denotes buffer regiori, no units.

$b(s) \quad$ Another constant of integration in " $s "$ space. atom-sec $/ \mathrm{cm}^{2}$ or atom-sec $/ \mathrm{cm}^{3}$

$c_{2}(r . s) \quad$ Transformed fission product concentration in coating. atom-sec/ $/ \mathrm{cm}^{3}$

$\mathrm{C}_{2}(\mathrm{r} . \mathrm{l}) \quad$ Fission product concentration in cuating as a function of position and time. atoms $/ \mathrm{cm}^{3}$

$C_{1}(1) \quad$ Total fission product concentration in source zone, atoms $/ \mathrm{cm}^{3}$

C!i (i) Mobile fission product corcentration in surce zone. atoms $/ \mathrm{cm}^{3}$

$\mathrm{C}_{1}^{0} \quad$ Initial fission jroduct concentration $\mathrm{C}_{1}^{*}(0)$ in source zone. atoms $/ \mathrm{cm}^{3}$

D Diffusion co 2 fficient for fission product migiation in coating. $\mathrm{cm}^{2} / \mathrm{sec}$

$D^{*} \quad$ Approximate diffusion coefficient when $T_{0}^{\prime}=1 / 6, \mathrm{cin} \mathrm{n}^{2} / \mathrm{sec}$

e The transiendental number. $2.7 \mathrm{i} 82 \ldots$ no units

f. Fraction of mobile fission product ia the coated particle. $(1-f)$ is the trapped fraction. no units

$f_{j}(L . T) \quad F u n c t i o n s$ of $L$ and $T, j=1.2 .3$. and 4. with charac!eristic roots $\alpha_{n}$, no units

$f(s) \quad$ Laplace transform of $F(t),[F(t)$ units $] \times[$ sec $]$

$F(t) \quad$ A general function of time. arbitrary units

FR Fraction of total fission product reieased, no units

$h \quad$ Geometric-partition parameter, $\left(a^{\prime} \phi\right)^{-1}$, for depleting systems, $(\mathrm{cm})^{-1}$

$h^{\prime} \quad$ Film coefficient for mass transier, $\mathrm{cm} / \mathrm{sec}$

H Film-or evaporation-diffusion coefficient ratio, $h^{\prime} / D .(\mathrm{cm})^{-1}$ 
k,

m
The imaginary number $(-1)^{1 / 2}$, no units

An index, no units

Diffusive flux as a function of $r$ and $t$, atoms $/ \mathrm{cm}^{2} \cdot \mathrm{sec}$

Spherical coordinate partition paramcter, $3 / \phi$. no units

As a subsciript, denotes kerne. regior, no units

Henry's law constant in terms of concentration, nu urits

.tenry's law constant ir. terms of pressure, atm $\cdot \mathrm{cm}^{3} /$ atom

Coating thickness, $b$ a, cm

Correlation parameter. $t_{\ell} l$, for depleting systems, no units

Correlation parameter, $H Q$, for film systems, nc units

Slope of experimental FR.vs-plot, $(\mathrm{sec})^{-1}$

Slope of theoretical FR/f-vs-T plot, no units

Amount of fission product in source regioli at any time, $t$, atoms

Amount of mobile fission product in source region, atoms

Initial amount of fission product in source region, atoms

Ultimate amount of fission product released, $\mathbf{M}_{1}^{0}$, atoms

Index, $n=1,2,3, \ldots$ no units

Partial density at inobile fission product in source region as a function of time, atoms $/ \mathrm{cm}^{3}$ - pore volume

$Q\left(a, k, \alpha_{r_{1}}^{\prime}\right)$ Modulus, or common denominator of transport functicns in spherical coordinates, no units

$\mathbf{r}$

General radial position in soherical coordinates, $\mathrm{cm}$

$\mathbf{R} \quad$ Ratio of di: ensions, a $\ell$, no units

S The Lapiace transform variable. $(\mathrm{sec})^{-1}$

RT Gas constant-2osolute temperature ratio, atm- $\mathrm{cm}^{3} /$ aton

Laboratory anned time, hr or s.ec

i A par:icular time t, hr o: s,e:;

io Experimental breakthrough time, hr or sec

$\mathrm{t}^{\prime} \quad$ Displaced anneal time, hi or sec

$t_{0}^{\prime} \quad$ Displaced initial time, hr or sec

T Reduced time, $\mathrm{Dt} / \ell^{2}$, no units

$T_{0} \quad$ Reduced breakthrougt، time, $D t_{0} / l^{2}$, no units

$T_{0}^{\prime} \quad$ Special reduced breakthrough time on depletion c!irve, ilo units

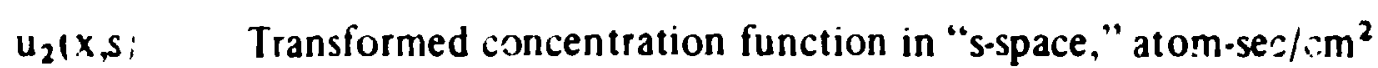

$\mathrm{U}_{2}(\mathrm{x} . t) \quad$ Transformed concentration function, $\mathrm{rC}_{2}(\mathrm{r}, \mathrm{t})$, in " $\mathrm{t}$-space," atoms $/ \mathrm{cm}^{2}$

$V_{\mathrm{J}} \quad$ Volume decorated with various sutscripts $\mathrm{j}, \mathrm{cm}^{3}$ 


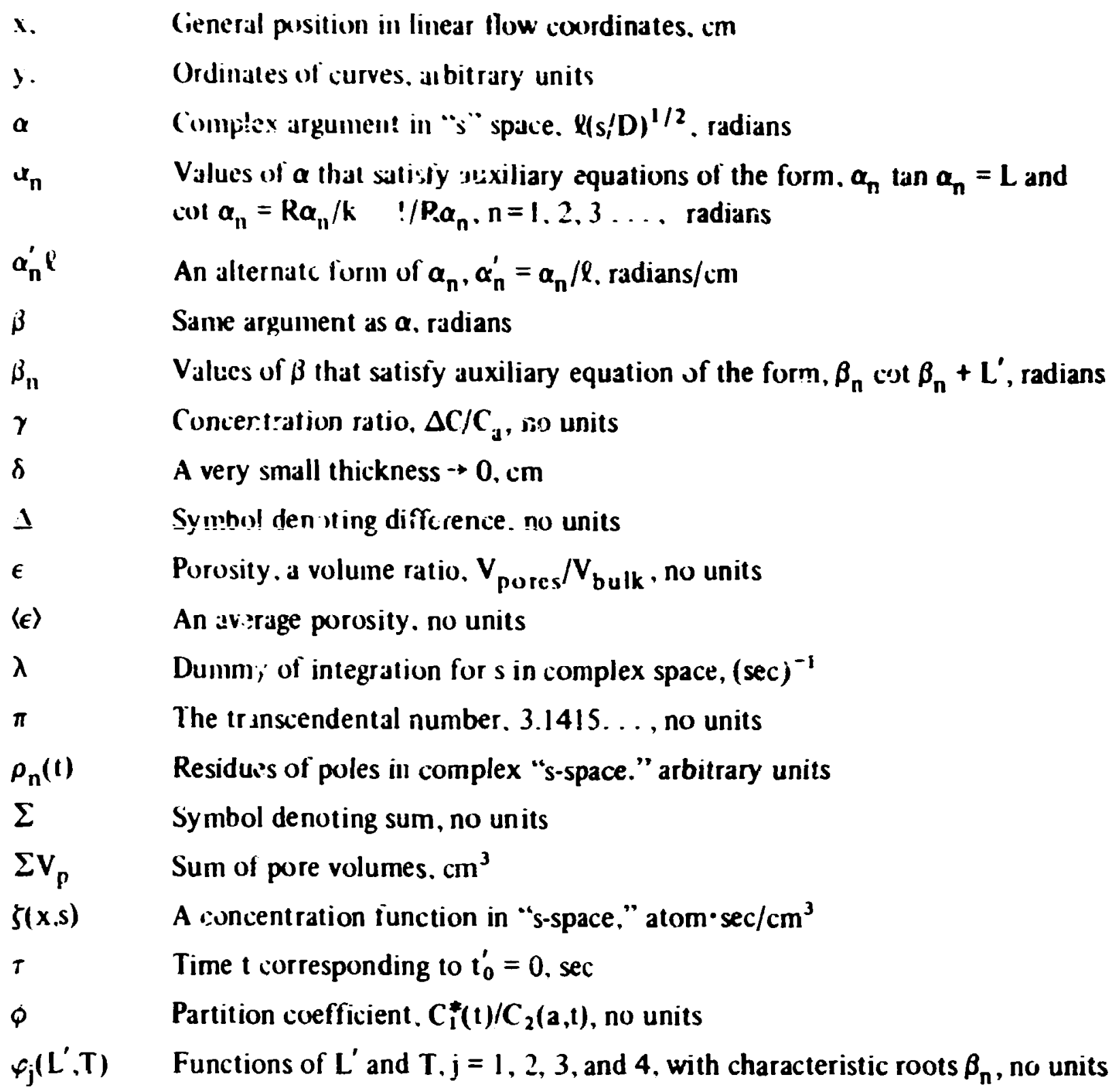

\section{REF'ERENCES}

1. H. J. de Nordwall, J. O. Kolb, F. F. Dyer, and W. J. Martir, pp. 172-75 in "Fission Prrduct Behavior in the Coolant Circuit of the Peach Bottom HTGR," GCR Programs Progr. Rept. Dec. 31, 1972, ( jAER Report ORNL-4911 (March 1974). Available from N.T.I.S. U.S. Dept. of Commerc2, 5?:5 Port l oyal Rd.. Springfield, Va 22151.

2. P. R. Rowland, W. E. Browning, and M. Carlyle, "Bohovior of Icdilı? Isotopes in a High Temperature Gas Reactor Coolant Circuit," Working Paper Annex of: Control of Iodint in the Nuclear Industry, Tech. Rpt. Ser. Nc. 148, International Atomic Eneigy A zency, Vienna (1973): pp. 57-71. Although this paper stre:ses iodine trar sport, profiles for displaced cesium along the heat sxchanger are shown in Fig. 6.

3. T. D. Gulden, J. L. Sco:t. and C. Moreau, Present Thoriurt-Cycle Fue' Concepts and Performance Limitaiions, USAEC Report GA-A 12877 (Feb. 1, 1974). Reprint of paper distributed at the American Nuclear Society Topical Miteting on GCRs, Gatlinburg. Tenn., May 8-10, 1974.

7. C. Mancini. R. Del Beccarn, and F. P. O. Ashworth, Safety Aspects of HTRs, Paper No. 80, British Nuclear Energy Society international Conference on Nuclear Fuel Performance, London, England, Oct. 15- 9, 19;3. This papar presents BISO vs TRISO (silicon carbide) argurments irom tire stancipoint of sateiy.

5. G. '. Raines, C. W. Townley, S. D. Beck, and W. H. Goldthwaite, A Method for the Study and Correlation of Fission-Gas-Release Behavior of Fuel Materials Dur:ig Irradiation, Appendix E. !ISAEC Report BMI-1548 (Oct. 4. 1961). Available from USAEC Technical Information Center, P.íi. Box 6... Odk Ridge, Tenn. 37830. 
6. R. W. Dunlap and T. D. Gulden, "Diffusion Model for Release of Fission Products frum Coated Fuels," Nucl. Sti. Eng. 32, $407 \quad 17$ (1968).

7. H. Walther, "Calculation of Releare of Fission Products from Coated Fuel Particles," Nukleonik $11(4), 171.78(1968)$.

8. J. H. N'iman, P. Winchell, J. M. Dixon, B. W. Roos, and R. F. Kurts, "Spheres: Diffusit n-Controlled Fission Product Release and Abs?rption," in Advances in Chemistry Series No. 93 "Radionuclides in the Environinen:," Reprint by Am. Chem. Soc. (1970), pp. 13-34.

9. (a) M. T. Morgan and R. L. Towns, Teikniques and Apparatus for inspernion, Handling, cand Annealing Highly Radioactive Fuel Microspheres, ORNL-TM-2495 (March 1969). (b) An updated version of this material is now in preparation and will appear in the following form: M. T. Morgan, Fission Product Release from Coated Particles During Postirradiation Anneals, OR.NL-TM-4539.

10. R. B. Evans III, J. L. Rutherford, and R. B. Perez, "Recoil of Fission Products. II In Heterogeneous Carbon Struriures," J. Appi. Phyjs. 39, 3253-67 (1968). Reference is made here to the structure of, an $\mathrm{J}$ the results for, a medium-density pyrocarbon - actually an HTI, Fig. 1.

11. H. S. Carslaw and J. C. Jaeger, Conduction of Hea: in Solids, 2nd ed., pp. 347-52, Uxiord University Press, New York, 1959.

12. H. S. Carslaw and J. C. Jaeger, Conduction of Heat in Solids, 2nd ed., p. 350, Eq. (24), Oxford University Press, New York, 1959.

13. H. S. Carslaw and J. C. Jaeger, Conduction of Heat in Sodids, 2nd eu., p. 128. Eq. (8), Oxford University Piess. New York. 1959.

14. j. C. Jaeger and Martha Clarke, "Numerical Results for Some Problems on Conduction of Heat in Slabs with Various Surface Conditions," Phil. Mag. 38, 504-15 (1947).

15. H. S. Carslaw and J. C. Jaeger, Condiction of Heat in Solids, 2nd ed., pp. 121, 123-25, Oxford University Press, New York. 1959.

16. J. Crank. The Mathematics of Diffusion, ist ed., pp. 47-48 and 95-96, Oxford University Press, New York, 1957.

17. K. W. Baurmann, Experimental Determination of Radial Concentration Profiles of S. lid Fission Products and Heavy Metals in Outer Coatings of Spherical Fuel Particles for High Te:nperature Reactors, Jül-639-R.G. (February 1970). Availability: Central Library of the Jülich Nuclear Research Center, Jülich, West Germany.

18. B T. Bradbury, J. T. Demant, and Mrs. P. M. Martin. "Solid Fission.-Products in liradiated Uranium Dioxide," Prũ Brit. Ceram. Soc. No. 7, 311-29(1967). 